QUARTERLY OF APPLIED MATHEMATICS

VOLUME LXIV, NUMBER 3

SEPTEMBER 2006, PAGES 561-591

S $0033-569 X(06) 01030-3$

Article electronically published on July 18, 2006

\title{
ASYMPTOTIC BEHAVIOUR OF SOLUTIONS OF LUBRICATION PROBLEM IN A THIN DOMAIN WITH A ROUGH BOUNDARY AND TRESCA FLUID-SOLID INTERFACE LAW
}

\author{
BY
}

MAHDI BOUKROUCHE (Laboratory of Mathematics, University of Saint-Etienne, EA 3989, 23 rue Dr. Paul Michelon, 42023 Saint-Etienne, France)

AND

IONEL CIUPERCA (Institut Camille Jordan, Université Lyon 1, UMR 5208, France)

\begin{abstract}
We study the asymptotic behavior of the solution of a Stokes flow in a thin domain, with a thickness of order $\varepsilon$, and a rough surface. The roughness is defined by a quasi-periodic function with period $\varepsilon$. We suppose that the flow is subject to a Tresca fluid-solid interface condition. We prove a new result on the lower-semicontinuity for the two-scale convergence, which allows us to obtain rigorously the limit problem and to establish the uniqueness of its solution.
\end{abstract}

1. Introduction. We consider in this paper a Stokes system with given exterior forces in a three-dimensional thin domain where the thickness is of order $\lambda \varepsilon$, for $\lambda>0$ a fixed constant and $\varepsilon$ a small parameter which will tend to zero. The boundary of this thin domain consists of three parts: the bottom, the lateral part, and the top surface. This research is motivated by a free boundary problem from lubrication theory where the domain of the flow is usually very thin and the roughness of the boundary strongly affects the flow. Moreover, in many problems of lubrication theory the widely assumed no-slip conditions when the fluid has the same velocity as the surrounding solid boundary seem not respected since the shear rate becomes too high [1, 2]; see also [3, 4], [5], 6], [7. We consider then that on the bottom, the normal velocity is equal to zero but the tangential velocity is unknown and satisfies Tresca-type fluid-solid boundary conditions, with friction coefficient a given positive function. On the lateral and the top parts, we have adhering boundary conditions. The top surface is assumed to be rough with a roughness defined by a quasi-periodic function with period $\varepsilon$.

Received January 27, 2006.

2000 Mathematics Subject Classification. Primary 35R35, 35J85, 78M35, 78M40, 74K35.

Key words and phrases. Free boundary problem, Lubrication, Rough boundary, Tresca fluid-solid conditions, Homogenization, Lower-semicontinuity for the two-scale convergence, Reynolds equation.

E-mail address: Mahdi.Boukrouche@univ-st-etienne.fr

E-mail address: ciuperca@math.univ-lyon1.fr 
The weak form of the problem is a variational inequality [8], see also [3], where the roughness of the top surface was neglected. We use the same scaling as in [9], where only the adhering boundary conditions are considered. See also [10, [11, [12 for related work.

The main difficulty here is to pass to the two-scale limit in the variational inequality, due to the term coming from the Tresca fluid-solid boundary conditions. This difficulty induces us to prove a needed result of lower-semicontinuity for the two-scale convergence, using some results on the subdifferential and regularization of convex functions.

This article is organized as follows. In Section 2 we give a precise strong and weak formulation of the considered problem, and recall from [3] the existence and uniqueness of solutions, which are the velocity and the pressure of the flow. In Section 3 we establish some estimates, independent of the parameter $\varepsilon$, for the velocity and the pressure. In Section 4 we prove some convergence results. In Section 5 we establish a first relation between the two-scale limit of the velocity and the pressure. In Section 6] we obtain the variational inequality between the two-scale limit of the velocity and the pressure, using the result of lower-semicontinuity for the two-scale convergence. In Section 7 we study the limit problem and obtain the uniqueness of its solution. The needed result of lower-semicontinuity for the two-scale convergence is proved in the last part of this work, Section 8

2. Formulation of the problem. Let $\omega$ be an open set in $\mathbb{R}^{2}$ with Lipschitz boundary and $Y=[0,1]^{2}$ the basic cell in $\mathbb{R}^{2}$. We consider a smooth function $(z, y) \mapsto h(z, y)$, such that $h \in \mathcal{C}^{2}\left(\omega \times \mathbb{R}^{2}\right)$,

$$
\left.\begin{array}{l}
h \text { and } \frac{\partial h}{\partial y_{i}} \text { for } i=1,2 \quad \text { are periodic in } y, \\
\exists a, b>0 \quad a \leq h(z, y) \leq b \quad \forall(z, y) \in \omega \times Y .
\end{array}\right\}
$$

We set

$$
h_{M}=\max _{(z, y) \in \omega \times Y} h(z, y), \quad h_{m}=\min _{(z, y) \in \omega \times Y} h(z, y),
$$

and we define the domains

$$
\left.\Omega_{M}=\omega \times\right] 0, h_{M}\left[, \quad \Omega_{m}=\omega \times\right] 0, h_{m}[.
$$

Let $\varepsilon$ be a small parameter, related to the roughness wavelength and also to the thickness of the gap between the surfaces $z_{3}=0$ and $z_{3}=\lambda \varepsilon h\left(z, \frac{z}{\varepsilon}\right)$, such that the domain occupied by the fluid is

$$
\Omega^{\varepsilon}=\left\{\left(z, z_{3}\right) \in \mathbb{R}^{3}: \quad z \in \omega, \quad 0<z_{3}<\lambda \varepsilon h^{\varepsilon}(z)\right\}
$$

where

$$
h^{\varepsilon}(z)=h\left(z, \frac{z}{\varepsilon}\right), \quad z \in \omega,
$$

and $\lambda>0$ is a fixed constant.

Let $\Gamma^{\varepsilon}$ be the boundary of $\Omega^{\varepsilon}$. We have $\Gamma^{\varepsilon}=\bar{\omega} \cap \bar{\Gamma}_{1}^{\varepsilon} \cap \bar{\Gamma}_{L}^{\varepsilon}$, where $\Gamma_{L}^{\varepsilon}$ is the lateral boundary, $\Gamma_{1}^{\varepsilon}$ is the upper surface defined by $z_{3}=\lambda \varepsilon h^{\varepsilon}(z)$, and $\omega$ is the bottom of the fluid domain defined by $z_{3}=0$. 
We need in the following to transform the domain $\Omega^{\varepsilon}$ into

$$
\Omega_{\varepsilon}=\left\{\left(x, x_{3}\right) \in \mathbb{R}^{3}: \quad x \in \omega, \quad 0<x_{3}<h^{\varepsilon}(x)\right\}
$$

using the scaling

$$
x=z \quad \text { and } \quad x_{3}=\frac{z_{3}}{\lambda \varepsilon},
$$

and also to transform the domain $\Omega_{\varepsilon}$ into $\left.\Omega=\omega \times\right] 0,1[$ using the scaling

$$
t=x \quad \text { and } \quad t_{3}=\frac{x_{3}}{h^{\varepsilon}} .
$$

Let $s^{0}=\left(s_{1}^{0}, s_{2}^{0}\right) \in \mathbb{R}^{2}$ be the velocity of the lower surface and $g$ be a given function in $\left(H^{\frac{1}{2}}\left(\partial \Omega_{m}\right)\right)^{3}$ which does not depend on $\varepsilon$, such that

$$
\begin{gathered}
g\left(x, h_{m}\right)=0, \quad g_{k}(x, 0)=s_{k}^{0} \quad \text { for } k=1,2 \text { and } g_{3}=0 \text { on } \partial \Omega_{m}, \\
\int_{\partial \Omega_{m}} g \cdot n d \sigma=0 .
\end{gathered}
$$

We consider the Stokes system

$$
\frac{\partial \sigma_{i j}^{\varepsilon}}{\partial z_{j}}+f_{i}^{\varepsilon}=0 \quad \text { in } \quad \Omega^{\varepsilon},
$$

where

$$
\begin{gathered}
\sigma_{i j}^{\varepsilon}=-p^{\varepsilon} \delta_{i j}+2 \mu d_{i, j}\left(u^{\varepsilon}\right) \quad \text { with } \quad d_{i, j}\left(u^{\varepsilon}\right)=\frac{1}{2}\left(\frac{\partial u_{i}^{\varepsilon}}{\partial z_{j}}+\frac{\partial u_{j}^{\varepsilon}}{\partial z_{i}}\right), \\
\operatorname{div}\left(u^{\varepsilon}\right)=0 \quad \text { in } \quad \Omega^{\varepsilon},
\end{gathered}
$$

where $u^{\varepsilon}, p^{\varepsilon}, \mu$ are the velocity, the pressure and the viscosity of the fluid.

On the upper surface, which is assumed to be fixed, no slip condition is given, so that

$$
u^{\varepsilon}=0 \quad \text { on } \quad \Gamma_{1}^{\varepsilon} .
$$

The velocity is known and parallel to the $\omega$-plane,

$$
u^{\varepsilon}=g\left(z, \frac{z_{3}}{\lambda \varepsilon}\right) \quad \text { on } \quad \Gamma_{L}^{\varepsilon},
$$

extended by 0 on $\left.\Gamma_{L}^{\varepsilon}-\partial \omega \times\right] 0, \lambda \varepsilon h_{m}[$.

There is a no flux condition across $\omega$ so that

$$
u_{3}^{\varepsilon}=0 \quad \text { on } \quad \omega .
$$

The tangential velocity on $\omega$ is unknown and satisfies the Tresca friction law with a friction coefficient $k^{\varepsilon}[\underline{8}$,

$$
\left.\begin{array}{l}
\left|\sigma_{t}^{\varepsilon}\right|<k^{\varepsilon} \Rightarrow u_{t}^{\varepsilon}=s^{0} \\
\left|\sigma_{t}^{\varepsilon}\right|=k^{\varepsilon} \Rightarrow \exists r \geq 0 \text { such that } u_{t}^{\varepsilon}=s^{0}-r \sigma_{t}^{\varepsilon}
\end{array}\right\} \text { on } \omega,
$$

where $\mid$. $\mid$ denotes the $\mathbb{R}^{2}$ Euclidean norm. Here $n=\left(n_{1}, n_{2}, n_{3}\right)$ is the unit outward normal vector to $\Gamma^{\varepsilon}$, and

$$
\begin{gathered}
u_{n}^{\varepsilon}=u^{\varepsilon} \cdot n=u_{i}^{\varepsilon} n_{i} ; \quad u_{t_{i}}^{\varepsilon}=u_{i}^{\varepsilon}-u_{n}^{\varepsilon} n_{i}, \\
\sigma_{n}^{\varepsilon}=\left(\sigma^{\varepsilon} \cdot n\right) \cdot n=\sigma_{i j}^{\varepsilon} n_{i} n_{j} ; \quad \sigma_{t_{i}}^{\varepsilon}=\sigma_{i j}^{\varepsilon} n_{j}-\sigma_{n}^{\varepsilon} n_{i}
\end{gathered}
$$


are, respectively, the normal, the tangential velocity on $\Gamma^{\varepsilon}$, the components of the normal and the tangential stress tensor on $\Gamma^{\varepsilon}$.

Due to (2.6) it is known [13] (lemma 2.2, p.24) that there exists a function $G$ such that

$$
G \in\left(H^{1}\left(\Omega_{m}\right)\right)^{3}: \quad \operatorname{div} G=0 \quad \text { in } \Omega_{m}, \quad G=g \quad \text { on } \partial \Omega_{m}
$$

We still denote by $G$ its extension by zero to $\Omega_{\varepsilon}$. We set $G^{\varepsilon}$ such that

$$
G_{i}^{\varepsilon}\left(z, z_{3}\right)=G_{i}\left(z, \frac{z_{3}}{\lambda \varepsilon}\right) \quad \text { for } \quad i=1,2, \quad G_{3}^{\varepsilon}\left(z, z_{3}\right)=\lambda \varepsilon G_{3}\left(z, \frac{z_{3}}{\lambda \varepsilon}\right),
$$

which belongs to $\left(H^{1}\left(\Omega^{\varepsilon}\right)\right)^{3}$ and satisfies also $\operatorname{div} G^{\varepsilon}=0$ in $\Omega^{\varepsilon}$.

We set

$$
\begin{gathered}
K^{\varepsilon}=\left\{v \in\left(H^{1}\left(\Omega^{\varepsilon}\right)\right)^{3}: \quad v=G^{\varepsilon} \text { on } \Gamma_{L}^{\varepsilon} \cup \Gamma_{1}^{\varepsilon}, \quad v_{3}=0 \text { on } \omega\right\}, \\
K_{\operatorname{div}}^{\varepsilon}=\left\{v \in K^{\varepsilon}: \quad \operatorname{div} v=0 \text { in } \Omega^{\varepsilon}\right\} .
\end{gathered}
$$

For any three-dimensional vector $\varphi=\left(\varphi_{1}, \varphi_{2}, \varphi_{3}\right)^{T}$ we denote by $\varphi$ the projected bi-dimensional vector $\underline{\varphi}=\left(\underline{\varphi}_{1}, \underline{\varphi}_{2}\right)^{T}$. A formal application of (2.7)-(2.12) leads to the following variational problem [8]:

Problem 2.1. Find $u^{\varepsilon} \in K_{\mathrm{div}}^{\varepsilon}\left(\Omega^{\varepsilon}\right)$ and $p^{\varepsilon} \in L_{0}^{2}\left(\Omega^{\varepsilon}\right)$ such that

$$
\begin{gathered}
a\left(u^{\varepsilon}, \varphi^{\varepsilon}-u^{\varepsilon}\right)-\left(p^{\varepsilon}, \operatorname{div} \varphi^{\varepsilon}\right)+j\left(\varphi^{\varepsilon}\right)-j\left(u^{\varepsilon}\right) \\
\geq\left(f^{\varepsilon}, \varphi^{\varepsilon}-u^{\varepsilon}\right), \quad \forall \varphi^{\varepsilon} \in K^{\varepsilon}
\end{gathered}
$$

where

$$
\begin{gathered}
a(u, \varphi)=2 \mu \sum_{i, j=1}^{3} \int_{\Omega^{\varepsilon}} d_{i j}(u) d_{i j}(\varphi) d z d z_{3} \\
j(\varphi)=\int_{\omega} k^{\varepsilon}\left|\underline{\varphi}-s^{0}\right| d z, \quad\left(f^{\varepsilon}, \varphi\right)=\sum_{i=1}^{3} \int_{\Omega^{\varepsilon}} f_{i}^{\varepsilon} \varphi_{i} d z d z_{3} \\
(p, \operatorname{div} \varphi)=\sum_{i=1}^{3} \int_{\Omega^{\varepsilon}} p \frac{\partial \varphi_{i}}{\partial z_{i}} d z d z_{3} .
\end{gathered}
$$

Theorem 2.1. 3] Assume that $f^{\varepsilon}$ in $\left(L^{3}\left(\Omega^{\varepsilon}\right)\right)^{3}$ and the friction coefficient $k^{\varepsilon}$ is a nonnegative function in $L^{\infty}(\omega)$. There exists a unique $u^{\varepsilon}$ in $K_{\text {div }}^{\varepsilon}$ and there exists a unique $p^{\varepsilon}$ in $L_{0}^{2}\left(\Omega^{\varepsilon}\right)$, such that $\left(u^{\varepsilon}, p^{\varepsilon}\right)$ is a solution to Problem 2.1

3. Estimates of the velocity and pressure. Observe first that as $u^{\varepsilon}\left(z, \lambda \varepsilon h^{\varepsilon}(z)\right)=$ 0 , we can extend it by zero to the fixed domain $\Omega_{M}\left(\bar{u}=u\right.$ in $\Omega^{\varepsilon}$ and $\bar{u}=0$ in $\left.\Omega_{M}-\Omega_{\varepsilon}\right)$, such that Korn's inequality

$$
C_{1}\|\nabla \bar{u}\|_{L^{2}\left(\Omega_{M}\right)} \leq a(\bar{u}, \bar{u}) \quad\left(C_{1} \text { independent of } \varepsilon\right)
$$

holds. Moreover we can easily check that $C_{1}=\frac{1}{2 \mu}$. So we have also the same inequality for $u^{\varepsilon}$ in $\Omega^{\varepsilon}$.

Throughout this paper, for the sake of simplicity, the same notation will be used for a function defined on the three different domains $\Omega^{\varepsilon}, \Omega_{\varepsilon}$ and $\Omega$. 
We assume the following dependence of data on $\varepsilon$ :

$$
\varepsilon^{2} f^{\varepsilon}\left(z, z_{3}\right)=f\left(x, x_{3}\right), \quad \varepsilon k^{\varepsilon}(z)=k(x)
$$

with $f$ and $k$ independent of $\varepsilon, f \in\left(L^{2}\left(\Omega_{m}\right)\right)^{3}$ (extended by 0 on $\Omega^{\varepsilon}$ ) and $k \in L^{2}(\omega)$ with $k>0$ a.e. We set

$$
\begin{gathered}
\hat{K}^{\varepsilon}=\left\{v \in\left(H^{1}\left(\Omega_{\varepsilon}\right)\right)^{3} ; v=\left(G_{1}, G_{1}, 0\right) \text { on } \hat{\Gamma^{\varepsilon}}{ }_{L} \cap \hat{\Gamma}^{\varepsilon_{1}}, \quad v_{3}=0 \text { on } \omega\right\} \\
\text { with } \quad \hat{\Gamma}^{\varepsilon} L=\left\{\left(x, x_{3}\right): x \in \partial \omega, \quad 0 \leq x_{3} \leq h^{\varepsilon}(x)\right\} \\
\text { and } \hat{\Gamma}^{\varepsilon}{ }_{1}=\left\{\left(x, h^{\varepsilon}(x)\right): x \in \omega\right\} .
\end{gathered}
$$

Then passing to $\Omega_{\varepsilon}$, and dividing by $\lambda \varepsilon,(2.17)$ is equivalent to

$$
\begin{gathered}
\hat{a}\left(u^{\varepsilon}, \varphi^{\varepsilon}-u^{\varepsilon}\right)-\int_{\Omega_{\varepsilon}} p^{\varepsilon}\left(\frac{\partial \varphi_{1}^{\varepsilon}}{\partial x_{1}}+\frac{\partial \varphi_{2}^{\varepsilon}}{\partial x_{2}}+\frac{1}{\lambda \varepsilon} \frac{\partial \varphi_{3}^{\varepsilon}}{\partial x_{3}}\right) d x d x_{3} \\
+\frac{1}{\lambda \varepsilon^{2}}\left(\hat{j}\left(\varphi^{\varepsilon}\right)-\left(\hat{j}\left(u^{\varepsilon}\right)\right) \geq \frac{1}{\varepsilon^{2}} \int_{\Omega_{\varepsilon}} f \cdot\left(\varphi^{\varepsilon}-u^{\varepsilon}\right) d x d x_{3} \quad \forall \varphi^{\varepsilon} \in \hat{K}^{\varepsilon}\right.
\end{gathered}
$$

with the notation

$$
\begin{aligned}
\hat{a}\left(u^{\varepsilon}, \varphi^{\varepsilon}\right)= & \frac{\mu}{2} \sum_{i, j=1}^{2} \int_{\Omega_{\varepsilon}}\left(\frac{\partial u_{i}^{\varepsilon}}{\partial x_{j}}+\frac{\partial u_{j}^{\varepsilon}}{\partial x_{i}}\right)\left(\frac{\partial \varphi_{i}^{\varepsilon}}{\partial x_{j}}+\frac{\partial \varphi_{j}^{\varepsilon}}{\partial x_{i}}\right) d x d x_{3} \\
& +\mu \sum_{i=1}^{2} \int_{\Omega_{\varepsilon}}\left(\frac{1}{\lambda \varepsilon} \frac{\partial u_{i}^{\varepsilon}}{\partial x_{3}}+\frac{\partial u_{3}^{\varepsilon}}{\partial x_{i}}\right)\left(\frac{1}{\lambda \varepsilon} \frac{\partial \varphi_{i}^{\varepsilon}}{\partial x_{3}}+\frac{\partial \varphi_{3}^{\varepsilon}}{\partial x_{i}}\right) d x d x_{3} \\
& +\frac{2 \mu}{\lambda^{2} \varepsilon^{2}} \int_{\Omega_{\varepsilon}} \frac{\partial u_{3}^{\varepsilon}}{\partial x_{3}} \frac{\partial \varphi_{3}^{\varepsilon}}{\partial x_{3}} d x d x_{3}
\end{aligned}
$$

and

$$
\hat{j}\left(\varphi^{\varepsilon}\right)=\int_{\omega} k(x)\left|\underline{\varphi}^{\varepsilon}(x, 0)-s^{0}\right| d x .
$$

For any domain $D$ in $\mathbb{R}^{3}$ we introduce the Banach space

$$
V_{x_{3}}(D)=\left\{v \in L^{2}(D), \quad \frac{\partial v}{\partial x_{3}} \in L^{2}(D)\right\}
$$

with the norm

$$
\|v\|_{V_{x_{3}}(D)}=\|v\|_{L^{2}(D)}^{2}+\left\|\frac{\partial v}{\partial x_{3}}\right\|_{L^{2}(D)}^{2} .
$$

First we give the estimates of the velocity in the rescaled domain $\Omega_{\varepsilon}$; then we give the estimates of the velocity and pressure in the domain $\Omega$.

Proposition 3.1. The following estimates, for the velocity, are satisfied in $\Omega_{\varepsilon}$, with $C$ denoting a constant which does not depend on $\varepsilon$ :

$$
\left\|u_{i}^{\varepsilon}\right\|_{V_{x_{3}}\left(\Omega_{\varepsilon}\right)} \leq C, \quad\left\|\frac{\partial u_{i}^{\varepsilon}}{\partial x_{j}}\right\|_{L^{2}\left(\Omega_{\varepsilon}\right)} \leq \frac{C}{\lambda \varepsilon}, \quad 1 \leq i \leq 3, \quad 1 \leq j \leq 2 .
$$

Proof. Taking $\varphi^{\varepsilon}=G^{\varepsilon}$ in (2.17) we obtain

$$
a\left(u^{\varepsilon}, u^{\varepsilon}\right)+j\left(u^{\varepsilon}\right) \leq a\left(u^{\varepsilon}, G^{\varepsilon}\right)+j\left(G^{\varepsilon}\right)-\left(f^{\varepsilon}, G^{\varepsilon}\right)+\left(f^{\varepsilon}, u^{\varepsilon}\right)
$$


but $j\left(G^{\varepsilon}\right)=j\left(s^{0}\right)=0$. Then using also the Korn inequality we have

$$
\begin{gathered}
2 \mu\left\|\nabla u^{\varepsilon}\right\|_{L^{2}\left(\Omega^{\varepsilon}\right)}^{2} \leq \mu\left\|\nabla u^{\varepsilon}\right\|_{L^{2}\left(\Omega^{\varepsilon}\right)}\left\|\nabla G^{\varepsilon}\right\|_{L^{2}\left(\Omega^{\varepsilon}\right)}+\left\|u^{\varepsilon}\right\|_{L^{2}\left(\Omega^{\varepsilon}\right)}\left\|f^{\varepsilon}\right\|_{L^{2}\left(\Omega^{\varepsilon}\right)} \\
+\left\|G^{\varepsilon}\right\|_{L^{2}\left(\Omega^{\varepsilon}\right)}\left\|f^{\varepsilon}\right\|_{L^{2}\left(\Omega^{\varepsilon}\right)} .
\end{gathered}
$$

From

$$
-u^{\varepsilon}\left(z, z_{3}\right)=\int_{z_{3}}^{\lambda \varepsilon h^{\varepsilon}(z)} \frac{\partial u^{\varepsilon}}{\partial z_{3}}(z, \xi) d \xi
$$

we have

$$
\left|u^{\varepsilon}\left(z, z_{3}\right)\right|^{2} \leq\left(\int_{0}^{\lambda \varepsilon h^{\varepsilon}(z)}\left|\frac{\partial u^{\varepsilon}}{\partial z_{3}}(z, \xi)\right| d t\right)^{2} \leq\left(\lambda \varepsilon h_{M}\right)\left(\int_{0}^{\lambda \varepsilon h^{\varepsilon}(z)}\left|\frac{\partial u^{\varepsilon}}{\partial z_{3}}(z, \xi)\right|^{2} d \xi\right)
$$

so

$$
\begin{aligned}
\int_{\Omega^{\varepsilon}}\left|u^{\varepsilon}\left(z, z_{3}\right)\right|^{2} & \leq \lambda \varepsilon h_{M} \int_{0}^{\lambda \varepsilon h^{\varepsilon}(z)} \int_{\Omega^{\varepsilon}}\left|\frac{\partial u^{\varepsilon}}{\partial z_{3}}(z, \xi)\right|^{2} d z d z_{3} d \xi \\
& \leq \lambda^{2} \varepsilon^{2} h_{M}^{2}\left\|\frac{\partial u^{\varepsilon}}{\partial z_{3}}\right\|_{L^{2}\left(\Omega^{\varepsilon}\right)}^{2}
\end{aligned}
$$

thus

$$
\left\|u^{\varepsilon}\right\|_{L^{2}\left(\Omega^{\varepsilon}\right)} \leq \lambda \varepsilon h_{M}\left\|\nabla u^{\varepsilon}\right\|_{L^{2}\left(\Omega^{\varepsilon}\right)} .
$$

So

$$
\begin{aligned}
\left\|f^{\varepsilon}\right\|_{L^{2}\left(\Omega^{\varepsilon}\right)}\left\|u^{\varepsilon}\right\|_{L^{2}\left(\Omega^{\varepsilon}\right)} & \leq\left(\frac{\lambda \varepsilon h_{M}}{\sqrt{\mu}} \cdot\left\|f^{\varepsilon}\right\|_{L^{2}\left(\Omega^{\varepsilon}\right)}\right)\left(\sqrt{\mu}\left\|\nabla u^{\varepsilon}\right\|_{L^{2}\left(\Omega^{\varepsilon}\right)}\right) \\
& \leq \frac{h_{M}^{2} \lambda^{2} \varepsilon^{2}}{2 \mu}\left\|f^{\varepsilon}\right\|_{L^{2}\left(\Omega^{\varepsilon}\right)}^{2}+\frac{\mu}{2}\left\|\nabla u^{\varepsilon}\right\|_{L^{2}\left(\Omega^{\varepsilon}\right)}^{2}
\end{aligned}
$$

and

$$
\mu\left\|\nabla u^{\varepsilon}\right\|_{L^{2}\left(\Omega^{\varepsilon}\right)}\left\|\nabla G^{\varepsilon}\right\|_{L^{2}\left(\Omega^{\varepsilon}\right)} \leq \frac{\mu}{2}\left\|\nabla u^{\varepsilon}\right\|_{L^{2}\left(\Omega^{\varepsilon}\right)}^{2}+\frac{\mu}{2}\left\|\nabla G^{\varepsilon}\right\|_{L^{2}\left(\Omega^{\varepsilon}\right)}^{2} .
$$

Then

$$
\mu\left\|\nabla u^{\varepsilon}\right\|_{L^{2}\left(\Omega^{\varepsilon}\right)}^{2} \leq \mu\left\|\nabla G^{\varepsilon}\right\|_{L^{2}\left(\Omega^{\varepsilon}\right)}^{2}+\left(\frac{1}{2 \mu}+\frac{\lambda^{2} h_{M}^{2} \varepsilon^{2}}{2 \mu}\right)\left\|f^{\varepsilon}\right\|_{L^{2}\left(\Omega^{\varepsilon}\right)}^{2} .
$$

Using now the dependence of $G^{\varepsilon}$ and $f^{\varepsilon}$ on $\varepsilon$, and using the Poincaré inequality, we deduce (3.4).

Proposition 3.2. The following estimates, for the velocity and the pressure, are satisfied in $\Omega$, with $C$ denoting a constant which does not depends on $\varepsilon$ :

$$
\begin{gathered}
\left\|\frac{\partial u_{i}^{\varepsilon}}{\partial t_{3}}\right\|_{L^{2}(\Omega)} \leq C \quad \text { for } \quad i=1,2,3, \\
\varepsilon\left\|\frac{\partial u_{i}^{\varepsilon}}{\partial t_{j}}\right\|_{L^{2}(\Omega)} \leq C \quad \text { for } \quad i=1,2,3 \quad j=1,2, \\
\varepsilon^{2}\left\|\frac{\partial p^{\varepsilon}}{\partial t_{i}}\right\|_{H^{-1}(\Omega)} \leq C, \quad i=1,2, \quad \varepsilon\left\|\frac{\partial p^{\varepsilon}}{\partial t_{3}}\right\|_{H^{-1}(\Omega)} \leq C .
\end{gathered}
$$


Proof. Using the change of variables (2.3) and (2.4) we have

$$
\begin{gathered}
\frac{D\left(x_{1}, x_{2}, x_{3}\right)}{D\left(t_{1}, t_{2}, t_{3}\right)}=\left(\begin{array}{ccc}
1 & 0 & 0 \\
0 & 1 & 0 \\
t_{3} \frac{\partial h^{\varepsilon}}{\partial t_{1}} & t_{3} \frac{\partial h^{\varepsilon}}{\partial t_{2}} & h^{\varepsilon}
\end{array}\right), \\
\frac{D\left(t_{1}, t_{2}, t_{3}\right)}{D\left(x_{1}, x_{2}, x_{3}\right)}=\left(\begin{array}{ccc}
1 & 0 & 0 \\
0 & 1 & 0 \\
-\frac{t_{3}}{h^{\varepsilon}} \frac{\partial h^{\varepsilon}}{\partial t_{1}} & -\frac{t_{3}}{h^{\varepsilon}} \frac{\partial h^{\varepsilon}}{\partial t_{2}} & \frac{1}{h^{\varepsilon}}
\end{array}\right) .
\end{gathered}
$$

As

$$
\left\|\frac{\partial h^{\varepsilon}}{\partial t_{j}}\right\|_{L^{\infty}(\omega)} \leq \frac{C}{\varepsilon} \quad \text { for } \quad 1 \leq j \leq 2,
$$

for $1 \leq i \leq 3$ and $1 \leq j \leq 2$, we deduce

$$
\begin{gathered}
\left\|\frac{\partial u_{i}^{\varepsilon}}{\partial t_{3}}\right\|_{L^{2}(\Omega)}^{2}=\int_{\Omega_{\varepsilon}}\left(h^{\varepsilon} \frac{\partial u_{i}^{\varepsilon}}{\partial x_{3}}\right)^{2} \frac{1}{h^{\varepsilon}} d x d x_{3} \leq h_{M}\left\|\frac{\partial u_{i}^{\varepsilon}}{\partial x_{3}}\right\|_{L^{2}\left(\Omega_{\varepsilon}\right)}^{2}, \\
\left\|\frac{\partial u_{i}^{\varepsilon}}{\partial t_{j}}\right\|_{L^{2}(\Omega)}^{2}=\int_{\Omega_{\varepsilon}}\left(\frac{\partial u_{i}^{\varepsilon}}{\partial x_{j}}+t_{3} \frac{\partial h^{\varepsilon}}{\partial x_{j}} \frac{\partial u_{i}^{\varepsilon}}{\partial x_{3}}\right)^{2} \frac{1}{h^{\varepsilon}} d x d x_{3} \\
\leq C\left(\left\|\frac{\partial u_{i}^{\varepsilon}}{\partial x_{j}}\right\|_{L^{2}\left(\Omega_{\varepsilon}\right)}^{2}+\frac{1}{\varepsilon^{2}}\left\|\frac{\partial u_{i}^{\varepsilon}}{\partial x_{3}}\right\|_{L^{2}\left(\Omega_{\varepsilon}\right)}^{2}\right),
\end{gathered}
$$

so from (3.10) and (3.4) we deduce (3.6), and from (3.9), (3.11) and (3.4) we deduce (3.7).

To prove (3.8) we rewrite (2.17) with $\varphi^{\varepsilon}=u^{\varepsilon} \pm \psi$ for all $\psi \in\left(H_{0}^{1}\left(\Omega^{\varepsilon}\right)\right)^{3}$; thus

$$
\int_{\Omega^{\varepsilon}} p^{\varepsilon} \operatorname{div} \psi d z d z_{3}=a\left(u^{\varepsilon}, \psi\right)-\left(f^{\varepsilon}, \psi\right) \quad \forall \psi \in\left(H_{0}^{1}\left(\Omega^{\varepsilon}\right)\right)^{3} .
$$

On the other hand, the change of variables (2.3) and (2.4) gives

$$
\frac{D\left(z_{1}, z_{2}, z_{3}\right)}{D\left(t_{1}, t_{2}, t_{3}\right)}=\left(\begin{array}{ccc}
1 & 0 & 0 \\
0 & 1 & 0 \\
\lambda \varepsilon t_{3} \frac{\partial h^{\varepsilon}}{\partial t_{1}} & \lambda \varepsilon t_{3} \frac{\partial h^{\varepsilon}}{\partial t_{2}} & \lambda \varepsilon h^{\varepsilon}
\end{array}\right)
$$

and

so

$$
\frac{D\left(t_{1}, t_{2}, t_{3}\right)}{D\left(z_{1}, z_{2}, z_{3}\right)}=\left(\begin{array}{ccc}
1 & 0 & 0 \\
0 & 1 & 0 \\
-\frac{t_{3}}{h^{\varepsilon}} \frac{\partial h^{\varepsilon}}{\partial t_{1}} & -\frac{t_{3}}{h^{\varepsilon}} \frac{\partial h^{\varepsilon}}{\partial t_{2}} & \frac{1}{\lambda \varepsilon h^{\varepsilon}}
\end{array}\right)
$$

$$
\int_{\Omega^{\varepsilon}} p^{\varepsilon} \operatorname{div} \psi=\int_{\Omega} p^{\varepsilon}\left[\sum_{i=1}^{2}\left[\frac{\partial}{\partial t_{i}}\left(\lambda \varepsilon h^{\varepsilon} \psi_{i}\right)+\frac{\partial}{\partial t_{3}}\left(-\lambda \varepsilon t_{3} \frac{\partial h^{\varepsilon}}{\partial t_{i}} \psi_{i}\right)\right]+\frac{\partial \psi_{3}}{\partial t_{3}}\right] .
$$

Taking $\psi=(0,0, \varphi)$ in (3.12) and (3.13) with $\varphi \in H_{0}^{1}(\Omega)$ we get

$$
\begin{gathered}
\int_{\Omega} p^{\varepsilon} \frac{\partial \varphi}{\partial t_{3}} d t d t_{3}=\frac{2 \mu}{\lambda \varepsilon} \int_{\Omega} \frac{\partial u_{3}^{\varepsilon}}{\partial t_{3}} \frac{\partial \varphi}{\partial t_{3}} \frac{1}{h^{\varepsilon}} d t d t_{3}-\frac{\lambda}{\varepsilon} \int_{\Omega} f_{3} \varphi h^{\varepsilon} d t d t_{3} \\
+\sum_{i=1}^{2} \int_{\Omega} \mu\left(\frac{\partial u_{i}^{\varepsilon}}{\partial t_{3}} \frac{1}{\lambda \varepsilon h^{\varepsilon}}+\frac{\partial u_{3}^{\varepsilon}}{\partial t_{i}}-\frac{t_{3}}{h^{\varepsilon}} \frac{\partial h^{\varepsilon}}{\partial t_{i}} \frac{\partial u_{3}^{\varepsilon}}{\partial t_{3}}\right)\left(\frac{\partial \varphi}{\partial t_{i}}-\frac{t_{3}}{h^{\varepsilon}} \frac{\partial h^{\varepsilon}}{\partial t_{i}} \frac{\partial \varphi}{\partial t_{3}}\right) \lambda \varepsilon h^{\varepsilon} d t d t_{3},
\end{gathered}
$$


so from (3.6)-(3.7) and (3.9) we deduce

$$
\left|\int_{\Omega} p^{\varepsilon} \frac{\partial \varphi}{\partial t_{3}}\right| \leq \frac{C}{\varepsilon}\|\varphi\|_{H_{0}^{1}(\Omega)} .
$$

Taking $\psi=\left(\frac{\varphi}{h^{\varepsilon}}, 0,0\right)$ in (3.12) and (3.13) with $\varphi \in H_{0}^{1}(\Omega)$ we obtain

$$
\begin{aligned}
& \int_{\Omega} p^{\varepsilon} \frac{\partial(\lambda \varepsilon \varphi)}{\partial t_{1}} d t d t_{3}-\int_{\Omega} p^{\varepsilon} \frac{\partial}{\partial t_{3}}\left(\lambda \varepsilon t_{3} \frac{\partial h^{\varepsilon}}{\partial t_{1}} \frac{\varphi}{h^{\varepsilon}}\right) d t d t_{3}=-\int_{\Omega} \frac{\lambda}{\varepsilon} f_{1} \varphi h^{\varepsilon} d t d t_{3} \\
& +2 \mu \int_{\Omega}\left[\frac{\partial u_{1}^{\varepsilon}}{\partial t_{1}}-\frac{t_{3}}{h^{\varepsilon}} \frac{\partial h^{\varepsilon}}{\partial t_{1}} \frac{\partial u_{1}^{\varepsilon}}{\partial t_{3}}\right]\left[\frac{\partial}{\partial t_{1}}\left(\frac{\varphi}{h^{\varepsilon}}\right)-\frac{t_{3}}{h^{\varepsilon}} \frac{\partial h^{\varepsilon}}{\partial t_{1}} \frac{1}{h^{\varepsilon}} \frac{\partial \varphi}{\partial t_{3}}\right] \lambda \varepsilon h^{\varepsilon} d t d t_{3} \\
& +\mu \int_{\Omega}\left[\frac{\partial u_{1}^{\varepsilon}}{\partial t_{2}}-\frac{t_{3}}{h^{\varepsilon}} \frac{\partial h^{\varepsilon}}{\partial t_{2}} \frac{\partial u_{1}^{\varepsilon}}{\partial t_{3}}+\frac{\partial u_{2}^{\varepsilon}}{\partial t_{1}}-\frac{t_{3}}{h^{\varepsilon}} \frac{\partial h^{\varepsilon}}{\partial t_{1}} \frac{\partial u_{2}^{\varepsilon}}{\partial t_{3}}\right] \\
& \times\left[\frac{\partial}{\partial t_{2}}\left(\frac{\varphi}{h^{\varepsilon}}\right)-\frac{t_{3}}{h^{\varepsilon}} \frac{\partial h^{\varepsilon}}{\partial t_{2}} \frac{1}{h^{\varepsilon}} \frac{\partial \varphi}{\partial t_{3}}\right] \lambda \varepsilon h^{\varepsilon} d t d t_{3} ;
\end{aligned}
$$

hence from (3.6), (3.7) and (3.9) we get

$$
\left|\int_{\Omega} p^{\varepsilon} \frac{\partial(\varepsilon \varphi)}{\partial t_{1}} d t d t_{3}-\int_{\Omega} p^{\varepsilon} \frac{\partial}{\partial t_{3}}\left(\varepsilon t_{3} \frac{\partial h^{\varepsilon}}{\partial t_{1}} \frac{\varphi}{h^{\varepsilon}}\right) d t d t_{3}\right| \leq \frac{C}{\varepsilon}\|\varphi\|_{H_{0}^{1}(\Omega)} .
$$

Now taking $\psi=\left(0,0, t_{3} \frac{\partial h^{\varepsilon}}{\partial t_{1}} \frac{\varphi}{h^{\varepsilon}}\right)$ in (3.12) and (3.13), with $\varphi \in H_{0}^{1}(\Omega)$, we get

$$
\begin{aligned}
& \int_{\Omega} p^{\varepsilon} \frac{\partial}{\partial t_{3}}\left(\frac{t_{3}}{h^{\varepsilon}} \frac{\partial h^{\varepsilon}}{\partial t_{1}} \varphi\right) d t d t_{3}=2 \mu \int_{\Omega} \frac{\partial u_{3}^{\varepsilon}}{\partial t_{3}} \frac{1}{\lambda \varepsilon h^{\varepsilon}} \frac{\partial}{\partial t_{3}}\left(\frac{t_{3}}{h^{\varepsilon}} \frac{\partial h^{\varepsilon}}{\partial t_{1}} \varphi\right) d t d t_{3} \\
& -\int_{\Omega} \frac{\lambda}{\varepsilon} f_{3} t_{3} \frac{\partial h^{\varepsilon}}{\partial t_{1}} \varphi d t d t_{3}+\mu \sum_{i=1}^{2} \int_{\Omega}\left[\frac{\partial u_{i}^{\varepsilon}}{\partial t_{3}} \frac{1}{\lambda \varepsilon h^{\varepsilon}}+\frac{\partial u_{3}^{\varepsilon}}{\partial t_{i}}-\frac{t_{3}}{h^{\varepsilon}} \frac{\partial h^{\varepsilon}}{\partial t_{i}} \frac{\partial u_{3}^{\varepsilon}}{\partial t_{3}}\right] \\
& \times\left[\frac{\partial}{\partial t_{i}}\left(\frac{t_{3}}{h^{\varepsilon}} \frac{\partial h^{\varepsilon}}{\partial t_{1}} \varphi\right)-\left(\frac{t_{3}}{h^{\varepsilon}} \frac{\partial h^{\varepsilon}}{\partial t_{i}}\right)\left(\frac{\partial \varphi}{\partial t_{3}} \frac{t_{3}}{h^{\varepsilon}} \frac{\partial h^{\varepsilon}}{\partial t_{1}}+\frac{1}{h^{\varepsilon}} \frac{\partial h^{\varepsilon}}{\partial t_{1}} \varphi\right)\right] \lambda \varepsilon d t d t_{3} .
\end{aligned}
$$

Then, from (3.6), (3.7) and (3.9), we easily obtain

$$
\left|\int_{\Omega} p^{\varepsilon} \frac{\partial}{\partial t_{3}}\left(\frac{t_{3}}{h^{\varepsilon}} \frac{\partial h^{\varepsilon}}{\partial t_{1}} \varphi\right) d t d t_{3}\right| \leq \frac{C}{\varepsilon^{2}}\|\varphi\|_{H_{0}^{1}(\Omega)} .
$$

So, from (3.15) and (3.16) we obtain (for $i=1$ but it is the same for $i=2$ )

$$
\left|\int_{\Omega} p^{\varepsilon} \frac{\partial \varphi}{\partial t_{i}} d t d t_{3}\right| \leq \frac{C}{\varepsilon^{2}}\|\varphi\|_{H_{0}^{1}(\Omega)} \quad \text { for } \quad i=1,2 .
$$

So from (3.14), (3.17) we deduce (3.8).

For the next sections we need the following notation: for any function $\psi$, we denote its extension from $\Omega_{\varepsilon}$ to $\Omega_{M}$ by

$$
\bar{\psi}=\left\{\begin{array}{lll}
\psi & \text { on } & \Omega_{\varepsilon} \\
0 & \text { on } & \Omega_{M}-\Omega_{\varepsilon} .
\end{array}\right.
$$


We define also the sets

$$
\begin{aligned}
B_{x} & \left.=\left\{\left(y, x_{3}\right), \quad y \in Y, \quad 0<x_{3}<h(x, y)\right\}, \quad Q_{M}=\omega \times Y \times\right] 0, h_{M}[, \\
Q & =\bigcup_{x \in \omega}\{x\} \times B_{x}=\left\{\left(x, y, x_{3}\right), \quad x \in \omega, \quad y \in Y, \quad 0<x_{3}<h(x, y)\right\}
\end{aligned}
$$

and the spaces

$$
\begin{gathered}
\mathcal{C}_{\#}^{\infty}\left(B_{x}\right)=\left\{\varphi \in \mathcal{C}^{\infty}\left(B_{x}\right), \quad \varphi \text { is } y \text {-periodic, } \varphi(y, h(x, y))=0\right\}, \\
H_{\#}^{1}(Y)=\left\{\varphi \in H^{1}(Y): \quad \varphi \text { is periodic }\right\}, \\
H_{0 \#}^{1}\left(B_{x}\right)=\left\{\varphi \in H^{1}\left(B_{x}\right): \varphi \text { is } y \text {-periodic and } \varphi(y, 0)=\varphi(y, h(x, y))=0\right\}, \\
H_{1 \#}^{1}\left(B_{x}\right)=\left\{\varphi \in H^{1}\left(B_{x}\right): \varphi \text { is } y \text {-periodic and } \varphi(y, h(x, y))=0\right\} .
\end{gathered}
$$

REMARK 3.1. We choose $p^{\varepsilon} \in L^{2}\left(\Omega^{\varepsilon}\right) / \mathbb{R}$ such that

$$
\int_{\Omega^{\varepsilon}} \frac{p^{\varepsilon}}{h^{\varepsilon}} d z d z_{3}=0, \quad \text { which implies } \int_{\Omega} p^{\varepsilon} d t d t_{3}=0 .
$$

We deduce from (3.8) and [14 that

$$
\varepsilon^{2}\left\|p^{\varepsilon}\right\|_{L^{2}(\Omega)} \leq C
$$

where $C$ is a constant independent of $\varepsilon$.

\section{Convergence results.}

Theorem 4.1. There exists $p \in L^{2}(\omega)$ with $\int_{\omega} p(x) d x=0$ such that the two-scale convergence holds in $\Omega_{M}$ for a subsequence of $\varepsilon$ which is still denoted by $\varepsilon$ :

$$
\varepsilon^{2} \overline{p^{\varepsilon}} \rightarrow \rightarrow \xi\left(x, x_{3}, y\right)=\left\{\begin{array}{lll}
p & \text { if } & x_{3}<h(x, y) \\
0 & \text { if } & x_{3}>h(x, y) .
\end{array}\right.
$$

Proof. We have

$$
\left\|p^{\varepsilon}\right\|_{L^{2}\left(\Omega_{\varepsilon}\right)}=\int_{\Omega}\left|p^{\varepsilon}\right|^{2} h^{\varepsilon} d t d t_{3} \leq h_{M}\left\|p^{\varepsilon}\right\|_{L^{2}(\Omega)}^{2}
$$

so

$$
\varepsilon^{2}\left\|\bar{p}^{\varepsilon}\right\|_{L^{2}\left(\Omega_{M}\right)} \leq C
$$

We can extract a subsequence such that $\varepsilon^{2} \bar{p}^{\varepsilon}$ two-scale converges to some $\xi$ in $L^{2}\left(\Omega_{M} \times[0,1]^{3}\right)$. We choose an arbitrary $\varphi$ in $\mathcal{D}\left(\Omega_{M}, \mathcal{C}_{\#}^{\infty}(Y)\right)$ with $\varphi=0$ for $x_{3} \leq h(x, y)$, and we get

$$
\int_{\Omega_{M}} \varepsilon^{2} \overline{p^{\varepsilon}} \varphi\left(x, \frac{x}{\varepsilon}, x_{3}\right) d x d_{3}=0 .
$$

Passing now to the scale limit, we obtain

$$
\int_{\Omega_{M}} \int_{Y} \xi \varphi\left(x, y, x_{3}\right) d y d x d_{3}=0
$$

so $\xi=0$ for $x_{3} \geq h(x, y)$. We denote by $p$ the restriction of $\xi$ for $x_{3} \leq h(x, y)$. Following [9], we prove that $\xi$ depends only on $x$ for $x_{3} \leq h(x, y)$. For any $\psi$ in $\mathcal{D}\left(\Omega_{M} ; \mathcal{C}_{\#}^{\infty}\left([0,1]^{3}\right)\right)$ with $\psi=0$ for $x_{3} \geq h(x, y)$, taking

$$
\psi^{\varepsilon}\left(x, x_{3}\right)=\psi\left(x, x_{3}, \frac{x}{\varepsilon}, \frac{x_{3}}{\varepsilon}\right),
$$


and $\left(u_{1}^{\varepsilon}, u_{2}^{\varepsilon}, u_{3}^{\varepsilon} \pm \psi^{\varepsilon}\right)$ as a test function in (3.2), we have

$$
\begin{array}{r}
\frac{1}{\lambda \varepsilon} \int_{\Omega_{\varepsilon}} p^{\varepsilon}\left(\frac{\partial \psi}{\partial x_{3}}+\frac{1}{\varepsilon} \frac{\partial \psi}{\partial y_{3}}\right) d x d x_{3}=\mu \sum_{i=1}^{2} \int_{\Omega_{\varepsilon}}\left[\frac{1}{\lambda \varepsilon} \frac{\partial u_{i}^{\varepsilon}}{\partial x_{3}}+\frac{\partial u_{3}^{\varepsilon}}{\partial x_{i}}\right]\left[\frac{\partial \psi}{\partial x_{i}}+\frac{1}{\varepsilon} \frac{\partial \psi}{\partial y_{i}}\right] \\
+\frac{2 \mu}{\lambda^{2} \varepsilon} \int_{\Omega_{\varepsilon}} \frac{\partial u_{3}^{\varepsilon}}{\partial x_{3}}\left(\frac{\partial \psi}{\partial x_{3}}+\frac{1}{\varepsilon} \frac{\partial \psi}{\partial y_{3}}\right) d x d x_{3}-\int_{\Omega_{\varepsilon}} \frac{f_{3}}{\varepsilon^{2}} \psi d x d x_{3} .
\end{array}
$$

Then extending $u^{\varepsilon}$ and $p^{\varepsilon}$ by zero to $\Omega_{M}$ and passing to the two-scale limit after multiplication by $\varepsilon^{4}$ we deduce

$$
\int_{[0,1]^{3}} \int_{\Omega_{M}} \xi \frac{\partial \psi}{\partial y_{3}} d x d x_{3} d y d y_{3}=0,
$$

so $p$ does not depend on $y_{3}$. To prove that $\xi$ does not depend on $x_{3}$, we take

$$
\psi^{\varepsilon}\left(x, x_{3}\right)=\psi\left(x, x_{3}, \frac{x}{\varepsilon}\right),
$$

with $\psi$ in $\mathcal{D}\left(\Omega_{M}, \mathcal{C}_{\#}^{\infty}(Y), \psi=0\right.$ for $x_{3} \geq h(x, y)$ and $\left(u_{1}^{\varepsilon}, u_{2}^{\varepsilon}, u_{3}^{\varepsilon} \pm \psi^{\varepsilon}\right)$ as a test function in (3.2). Then

$$
\begin{aligned}
\frac{1}{\lambda \varepsilon} \int_{\Omega_{\varepsilon}} p^{\varepsilon} \frac{\partial \psi}{\partial x_{3}} d x d x_{3}= & \mu \sum_{i=1}^{2} \int_{\Omega^{\varepsilon}}\left[\frac{1}{\lambda \varepsilon} \frac{\partial u_{i}^{\varepsilon}}{\partial x_{3}}+\frac{\partial u_{3}^{\varepsilon}}{\partial x_{i}}\right]\left[\frac{\partial \psi}{\partial x_{i}}+\frac{1}{\varepsilon} \frac{\partial \psi}{\partial y_{i}}\right] d x d x_{3} \\
& +\frac{2 \mu}{\lambda^{2} \varepsilon^{2}} \int_{\Omega_{\varepsilon}} \frac{\partial u_{3}^{\varepsilon}}{\partial x_{3}} \frac{\partial \psi}{\partial x_{3}} d z d z_{3}-\int_{\Omega_{\varepsilon}} \frac{f_{3}}{\varepsilon^{2}} \psi d x d x_{3} .
\end{aligned}
$$

Passing to the two-scale limit after multiplication by $\varepsilon^{3}$ we deduce

$$
\int_{Y} \int_{\Omega_{\varepsilon}} \xi \frac{\partial \psi}{\partial x_{3}} d x d x_{3} d y=0
$$

So $p$ does not depend on $x_{3}$. To prove that $p$ does not depend on $y_{i}$ for $i=1,2$ we take

$$
\psi^{\varepsilon}\left(x, x_{3}\right)=\psi\left(x, x_{3}, \frac{x}{\varepsilon}\right)
$$

with $\psi$ in $\mathcal{D}\left(\Omega_{M}, \mathcal{C}_{\#}^{\infty}(Y)\right)$, where $\psi=0$ for $x_{3} \geq h(x, y)$ and $\left(u_{1}^{\varepsilon} \pm \psi^{\varepsilon}, u_{2}^{\varepsilon}, u_{3}^{\varepsilon}\right)$ as a test function in (3.2), and we obtain

$$
\begin{gathered}
\int_{\Omega_{\varepsilon}} p^{\varepsilon}\left(\frac{\partial \psi}{\partial x_{1}}+\frac{1}{\varepsilon} \frac{\partial \psi}{\partial y_{1}}\right) d x d x_{3}=2 \mu \int_{\Omega_{\varepsilon}} \frac{\partial u_{1}^{\varepsilon}}{\partial x_{1}}\left(\frac{\partial \psi}{\partial x_{1}}+\frac{1}{\varepsilon} \frac{\partial \psi}{\partial y_{1}}\right) d x d x_{3} \\
+\mu \int_{\Omega_{\varepsilon}}\left(\frac{\partial u_{1}^{\varepsilon}}{\partial x_{2}}+\frac{\partial u_{2}^{\varepsilon}}{\partial x_{1}}\right)\left(\frac{\partial \psi}{\partial x_{2}}+\frac{1}{\varepsilon} \frac{\partial \psi}{\partial y_{2}}\right) d x d x_{3} \\
+\mu \int_{\Omega_{\varepsilon}}\left(\frac{1}{\lambda \varepsilon} \frac{\partial u_{1}^{\varepsilon}}{\partial x_{3}}+\frac{\partial u_{3}^{\varepsilon}}{\partial x_{1}}\right) \frac{1}{\lambda \varepsilon} \frac{\partial \psi}{\partial x_{3}} d x d x_{3}-\frac{1}{\varepsilon^{2}} \int_{\Omega_{\varepsilon}} f_{1} \psi d x d x_{3} .
\end{gathered}
$$

Passing to the two-scale limit after multiplication by $\varepsilon^{3}$ we deduce

$$
\int_{\Omega_{M}} \int_{Y} \xi \frac{\partial \psi}{\partial y_{1}} d y d x d x_{3}=0
$$


So $p$ does not depend on $y_{1}$. In the same manner $p$ does not depend on $y_{2}$. It remains to prove that $\int_{\omega} p d x=0$. Passing to the two-scale limit in the relation

$$
\int_{\Omega_{M}} \frac{\varepsilon^{2} p^{\varepsilon}}{h^{\varepsilon}} d x d x_{3}=0
$$

we find

$$
\int_{Q} \frac{p}{h} d y d x d x_{3}=0
$$

which gives the expected result and finishes the proof.

Theorem 4.2. There exists $u \in\left(L^{2}\left(\Omega_{M} ; H_{\#}^{1}(Y)\right)\right)^{3}$ with

$$
\frac{\partial u}{\partial x_{3}} \in\left(L^{2}\left(\Omega_{M} \times Y\right)\right)^{3},
$$

and there exists $v \in\left(L^{2}\left(\Omega_{M} ; H_{\#}^{1}\left([0,1]^{3}\right)\right)^{3}\right.$ such that the two-scale convergences hold, up to a subsequence of $\varepsilon$ denoted also by $\varepsilon$ :

$$
\begin{gathered}
\bar{u}_{i}^{\varepsilon} \rightarrow u_{i}, \quad \varepsilon \frac{\partial \bar{u}_{i}^{\varepsilon}}{\partial x_{j}} \rightarrow \frac{\partial u_{i}}{\partial y_{j}} \quad \text { for } \quad 1 \leq i \leq 3, \quad 1 \leq j \leq 2, \\
\frac{\partial \bar{u}_{i}^{\varepsilon}}{\partial x_{3}} \rightarrow \rightarrow \frac{\partial u_{i}}{\partial x_{3}}+\frac{\partial v_{i}}{\partial y_{3}} \quad \text { for } \quad 1 \leq i \leq 3 .
\end{gathered}
$$

Moreover

$$
\begin{gathered}
\frac{\partial u_{1}}{\partial y_{1}}+\frac{\partial u_{2}}{\partial y_{2}}+\frac{1}{\lambda} \frac{\partial u_{3}}{\partial x_{3}}=0 \quad \text { in } \quad B_{x} \quad \text { a.e. } x \in \omega \\
u_{i}=0 \text { for } x_{3} \geq h(x, y) \text { for } \quad i=1,2,3 \\
u_{3}=0 \text { for } x_{3}=0, \\
\bar{u}_{i}^{\varepsilon}(x, 0) \rightarrow \rightarrow u_{i}(x, 0), \quad \text { for } \omega \text { for } \quad 1 \leq i \leq 2 .
\end{gathered}
$$

We have also the following relation satisfied by the $y, x_{3}$-average of $u$ :

$$
\int_{\omega} \sum_{i=1}^{2} \frac{\partial \theta}{\partial x_{i}}\left(\int_{Y} \int_{0}^{h_{M}} u_{i} d y d x_{3}\right) d x=\int_{\partial \omega} \tilde{g}_{i} n_{i} \theta d \sigma \quad \text { for any } \theta \in H^{1}(\omega),
$$

where

$$
\tilde{g}_{i}(x)=\int_{0}^{h_{m}} g d x_{3}
$$

Proof. (4.3)-(4.4) follow from the estimate (3.4) using the two-scale convergence results [15, [16] as in [9]. For (4.5), note that $u^{\varepsilon}$ is divergence free in $\Omega_{\varepsilon}$, so

$$
\frac{\partial \overline{u_{1}^{\varepsilon}}}{\partial x_{1}}+\frac{\partial \overline{u_{2}^{\varepsilon}}}{\partial x_{2}}+\frac{1}{\lambda \varepsilon} \frac{\partial \overline{u_{3}^{\varepsilon}}}{\partial x_{3}}=0 .
$$

Multiplying by $\varepsilon$, passing to the two-scale limit in $\Omega_{M}$ and integrating on $y_{3}$ we easily obtain (4.5).

For (4.6), choosing $\varphi$ in $\mathcal{C}^{\infty}\left(\Omega_{M}, \mathcal{C}_{\#}^{\infty}(Y)\right)$ with $\varphi=0$ for $x_{3} \leq h(x, y)$, we get

$$
\int_{\Omega_{M}} \bar{u}_{i}^{\varepsilon} \varphi\left(x, \frac{x}{\varepsilon}, x_{3}\right) d x d_{3}=0 .
$$


Passing now to the two-scale limit for $i=1,2,3$, we obtain

$$
\int_{\Omega_{M}} \int_{Y} \bar{u}_{i} \varphi\left(x, y, x_{3}\right) d y d x d_{3}=0 .
$$

So $\bar{u}_{i}=0$ for $x_{3} \geq h(x, y)$, for $i=1,2,3$.

From (2.9), (2.11), and for all $\varphi$ in $L^{2}\left(\omega ; \mathcal{C}_{\#}(Y)\right)$ we have

$$
\int_{\Omega_{M}} \frac{\partial \overline{u_{3}^{\varepsilon}}}{\partial x_{3}} \varphi\left(x, \frac{x}{\varepsilon}\right) d x d_{3}=0 .
$$

Passing to the two-scale limit we obtain

$$
\int_{\Omega_{M}} \int_{[0,1]^{3}}\left(\frac{\partial u_{3}}{\partial x_{3}}+\frac{\partial v_{3}}{\partial y_{3}}\right) \varphi(x, y) d y d y_{3} d x d x_{3}=0 .
$$

As

and using Green's formula we deduce

$$
\int_{[0,1]^{3}} \frac{\partial v_{3}}{\partial y_{3}} \varphi(x, y) d y d y_{3}=0
$$

$$
\int_{\omega} \int_{Y} u_{3}(x, y, 0) \varphi(x, y) d y d x=0,
$$

which proves (4.7).

In order to prove (4.8) let us remark from (3.4) that $u_{i}^{\varepsilon}(x, 0)$ is bounded in $L^{2}(\omega)$. Then there exists $\xi_{i} \in L^{2}(\omega \times Y)$ such that

$$
u_{i}^{\varepsilon}(x, 0) \rightarrow \xi_{i}(x, 0) \text { in } \omega .
$$

Let us identify $\xi$. Passing to the two-scale limit in the obvious identity

$$
\int_{\Omega_{M}} \frac{\partial u_{i}^{\varepsilon}}{\partial x_{3}} \varphi\left(x, \frac{x}{\varepsilon}\right) d x d x_{3}=-\int_{\omega} u_{i}^{\varepsilon}(x, 0) \varphi\left(x, \frac{x}{\varepsilon}\right) d x \quad \text { for any } \varphi \in L^{2}\left(\omega ; \mathcal{C}_{\#}(Y)\right)
$$

we obtain

$$
\int_{\Omega_{M}} \int_{Y} \int_{0}^{1}\left(\frac{\partial u_{i}}{\partial x_{3}}+\frac{\partial v_{i}}{\partial y_{3}}\right) \varphi(x, y) d y d y_{3} d x d x_{3}=-\int_{\omega} \int_{Y} \xi_{i}(x, y) \varphi(x, y) d y d x .
$$

The term in $v_{i}$ vanishes, so by Green's identity we find

$$
\int_{\omega} \int_{Y} u_{i}(x, y, 0) \varphi(x, y) d y d x=\int_{\omega} \int_{Y} \xi_{i}(x, y) \varphi(x, y) d y d x
$$

for an arbitrary $\varphi$, so $\xi_{i}(x, y)=u_{i}(x, y, 0)$ for $1 \leq i \leq 3$, which ends the proof of (4.8).

To prove (4.9) we write

$$
\int_{\Omega_{M}}\left(\sum_{i=1}^{2} \frac{\partial \bar{u}_{i}^{\varepsilon}}{\partial x_{i}}+\frac{1}{\lambda \varepsilon} \frac{\partial \bar{u}_{3}^{\varepsilon}}{\partial x_{3}}\right) \theta(x) d x d x_{3}=0 \quad \forall \theta \in H^{1}(\omega)
$$

so by Green's formula we have

$$
\int_{\Omega_{M}}\left(\sum_{i=1}^{2} \overline{u_{i}^{\varepsilon}}\right) \frac{\partial \theta}{\partial x_{i}} d x d x_{3}=\int_{\omega} \int_{0}^{h_{M}}\left(\sum_{i=1}^{2} g_{i} n_{i}\right) \theta(x) d x d x_{3} .
$$

Passing to the two-scale limit we obtain (4.9). 
5. A first relation between $p$ and $u$. In the following we need to transform the domain $B_{x}$ into $\left.Y \times\right] 0,1[$ using the scaling

$$
\xi=y, \quad \xi_{3}=\frac{x_{3}}{h(x, \xi)} .
$$

For any function $\psi$ defined on $B_{x}$ we define $\hat{\psi}$ defined on $\left.Y \times\right] 0,1[$ by

$$
\hat{\psi}\left(\xi, \xi_{3}\right)=\psi\left(y, x_{3}\right) \text {. }
$$

We have

$$
\begin{gathered}
\frac{D\left(y_{1}, y_{2}, x_{3}\right)}{D\left(\xi_{1}, \xi_{2}, \xi_{3}\right)}=\left(\begin{array}{ccc}
1 & 0 & 0 \\
0 & 1 & 0 \\
\xi_{3} \frac{\partial h}{\partial \xi_{1}} & \xi_{3} \frac{\partial h}{\partial \xi_{2}} & h
\end{array}\right)=A=A\left(x, \xi, \xi_{3}\right), \\
\frac{D\left(\xi_{1}, \xi_{2}, \xi_{3}\right)}{D\left(y_{1}, y_{2}, x_{3}\right)}=\left(\begin{array}{ccc}
1 & 0 & 0 \\
0 & 1 & 0 \\
-\frac{\xi_{3}}{h} \frac{\partial h}{\partial \xi_{1}} & -\frac{\xi_{3}}{h} \frac{\partial h}{\partial \xi_{2}} & \frac{1}{h}
\end{array}\right)=B=B\left(\left(x, \xi, \xi_{3}\right) .\right.
\end{gathered}
$$

We will also use the following notation: $\mathcal{V}=\left\{v \in\left(\mathcal{C}^{1}(\bar{Q})\right)^{3}\right.$ such that $\frac{\partial v_{1}}{\partial y_{1}}+\frac{\partial v_{2}}{\partial y_{2}}+$ $\frac{1}{\lambda} \frac{\partial v_{3}}{\partial x_{3}}=0, \quad v$ is $y$-periodic, and $v=0$ for $x_{3}=0$ or $\left.x_{3}=h(x, y)\right\} . \mathcal{V}^{0}=\{v \in \mathcal{V}: v=0$ for $x \in \partial \omega\}$.

For any $x \in \omega$ we set $\mathcal{V}_{x}=\left\{v \in\left(\mathcal{C}^{1}\left(\bar{B}_{x}\right)\right)^{3}\right.$ such that $\frac{\partial v_{1}}{\partial y_{1}}+\frac{\partial v_{2}}{\partial y_{2}}+\frac{1}{\lambda} \frac{\partial v_{3}}{\partial x_{3}}=0, \quad v$ is $y$-periodic, $v=0$ for $x_{3}=0$ or $\left.x_{3}=h(x, y)\right\} . V_{0, x}=\left\{v \in\left(H_{0 \#}^{1}\left(B_{x}\right)\right)^{3}: \frac{\partial v_{1}}{\partial y_{1}}+\frac{\partial v_{2}}{\partial y_{2}}+\right.$ $\left.\frac{1}{\lambda} \frac{\partial v_{3}}{\partial x_{3}}=0\right\}$

We also define the matrices

$$
A_{\lambda}=\left(\begin{array}{ccc}
1 & 0 & 0 \\
0 & 1 & 0 \\
\lambda \xi_{3} \frac{\partial h}{\partial \xi_{1}} & \lambda \xi_{3} \frac{\partial h}{\partial \xi_{2}} & \lambda h
\end{array}\right), \quad B_{\lambda}=A_{\lambda}^{-1}=\left(\begin{array}{ccc}
1 & 0 & 0 \\
0 & 1 & 0 \\
-\frac{\xi_{3}}{h} \frac{\partial h}{\partial \xi_{1}} & -\frac{\xi_{3}}{h} \frac{\partial h}{\partial \xi_{2}} & \frac{1}{\lambda h}
\end{array}\right) .
$$

We have the following technical lemmas.

Lemma 5.1. For any $x \in \omega$ and any $\psi \in\left(L^{2}\left(B_{x}\right)\right)^{3}$ the following two assertions are equivalent:

$$
\begin{gathered}
\frac{\partial \psi_{1}}{\partial y_{1}}+\frac{\partial \psi_{2}}{\partial y_{2}}+\frac{1}{\lambda} \frac{\partial \psi_{3}}{\partial x_{3}}=0, \\
\operatorname{div}_{\xi, \xi_{3}}\left(h B_{\lambda} \hat{\psi}\right)=0 .
\end{gathered}
$$

Proof. The relation (5.1) is equivalent to

$$
\int_{B_{x}}\left(\sum_{k=1}^{2} \psi_{k} \frac{\partial \varphi}{\partial y_{k}}+\frac{1}{\lambda} \psi_{3} \frac{\partial \varphi}{\partial y_{3}}\right) d y_{1} d y_{2} d x_{3}=0 \quad \forall \psi \in \mathcal{D}\left(B_{x}\right) .
$$

Passing to $Y \times[0,1]$ this gives

$$
\int_{Y \times[0,1]} \hat{\psi}^{T} \cdot B_{\lambda}^{T} \cdot \nabla_{\xi, \xi_{3}} \hat{\varphi} h d \xi d \xi_{3}=0,
$$

which is equivalent to (5.2). 
Lemma 5.2. For any $x_{0} \in \omega$ and any $v \in \mathcal{V}_{x_{0}}$ there exists $\varphi \in \mathcal{V}$ such that $\varphi\left(x_{0}, y, x_{3}\right)=$ $v\left(y, x_{3}\right)$.

Proof. We set $\hat{v}\left(\xi, \xi_{3}\right)=v\left(\xi, h\left(x_{0}, \xi\right) \xi_{3}\right)$ defined in $Y \times[0,1]$. We choose $\hat{\varphi}\left(x, \xi, \xi_{3}\right)$ defined in $\omega \times Y \times[0,1]$ such that

$$
h(x, \xi) B_{\lambda}\left(x, \xi, \xi_{3}\right) \hat{\varphi}=h\left(x_{0}, \xi\right) B_{\lambda}\left(x_{0}, \xi, \xi_{3}\right) \hat{v},
$$

that is,

$$
\hat{\varphi}\left(x, \xi, \xi_{3}\right)=\frac{h\left(x_{0}, \xi\right)}{h(x, \xi)} A_{\lambda}\left(x, \xi, \xi_{3}\right) B_{\lambda}\left(x_{0}, \xi, \xi_{3}\right) \hat{v}\left(\xi, \xi_{3}\right) .
$$

Finally we set

$$
\varphi\left(x, y, x_{3}\right)=\hat{\varphi}\left(x, y, \frac{x_{3}}{h(x, y)}\right) .
$$

Since by hypothesis

$$
\frac{\partial v_{1}}{\partial y_{1}}+\frac{\partial v_{2}}{\partial y_{2}}+\frac{1}{\lambda} \frac{\partial v_{3}}{\partial x_{3}}=0
$$

this implies by Lemma 5.1 that

$$
\operatorname{div}_{\xi, \xi_{3}}\left(h\left(x_{0}, \xi\right) B_{\lambda}\left(x_{0}, \xi, \xi_{3}\right) \hat{v}\right)=0,
$$

so by (5.3) and again Lemma 5.1

$$
\frac{\partial \varphi_{1}}{\partial y_{1}}+\frac{\partial \varphi_{2}}{\partial y_{2}}+\frac{1}{\lambda} \frac{\partial \varphi_{3}}{\partial x_{3}}=0
$$

The remaining conditions on $\varphi$ are obvious.

We now have a first relation between $p$ and $u$.

Theorem 5.3. The limit pressure $p$ belongs to $H^{1}(\omega)$ and the following relation yields a.e. $x$ in $\omega$,

$$
\begin{gathered}
2 \mu \sum_{i, j=1}^{3} \int_{B_{x}} D_{i, j}^{B}(u) D_{i, j}^{B}(\psi) d y d x_{3}=-\sum_{i=1}^{2} \int_{B_{x}} \psi_{i} \frac{\partial p}{\partial x_{i}} d y d x_{3} \\
+\sum_{i=1}^{3} \int_{B_{x}} f_{i} \psi_{i} d y d x_{3} \quad \forall \psi \in \mathrm{V}_{0, x},
\end{gathered}
$$

where for $1 \leq i, j \leq 2$,

$$
D_{i, j}^{B}(u)=\frac{1}{2}\left(\frac{\partial u_{i}}{\partial y_{j}}+\frac{\partial u_{j}}{\partial y_{i}}\right), \quad D_{i, 3}^{B}(u)=\frac{1}{2}\left(\frac{1}{\lambda} \frac{\partial u_{i}}{\partial x_{3}}+\frac{\partial u_{3}}{\partial y_{i}}\right), \quad D_{3,3}^{B}(u)=\frac{1}{\lambda} \frac{\partial u_{3}}{\partial x_{3}} .
$$

Proof. We proceed as in [9]. From (3.12) passing to $\Omega_{\varepsilon}$ we obtain, dividing by $\lambda \varepsilon$,

$$
\begin{aligned}
& \int_{\Omega_{M}} p^{\varepsilon}\left(\sum_{i=1}^{2} \frac{\partial \bar{\psi}_{i}^{\varepsilon}}{\partial x_{i}}+\frac{1}{\lambda \varepsilon} \frac{\partial \bar{\psi}_{3}^{\varepsilon}}{\partial x_{3}}\right)=\frac{\mu}{2} \sum_{i, j=1}^{2} \int_{\Omega_{M}}\left(\frac{\partial \bar{u}_{i}^{\varepsilon}}{\partial x_{j}}+\frac{\partial \bar{u}_{j}^{\varepsilon}}{\partial x_{i}}\right)\left(\frac{\partial \bar{\psi}_{i}^{\varepsilon}}{\partial x_{j}}+\frac{\partial \bar{\psi}_{j}^{\varepsilon}}{\partial x_{i}}\right) \\
& +\sum_{i=1}^{2} \mu \int_{\Omega_{M}}\left(\frac{1}{\lambda \varepsilon} \frac{\partial \bar{u}_{i}^{\varepsilon}}{\partial x_{3}}+\frac{\partial \overline{u_{3}^{\varepsilon}}}{\partial x_{i}}\right)\left(\frac{1}{\lambda \varepsilon} \frac{\partial \bar{\psi}_{i}^{\varepsilon}}{\partial x_{3}}+\frac{\partial \bar{\psi}_{3}^{\varepsilon}}{\partial x_{i}}\right)+\frac{2 \mu}{\lambda^{2} \varepsilon^{2}} \int_{\Omega_{M}} \frac{\partial \overline{u_{3}^{\varepsilon}}}{\partial x_{3}} \frac{\partial \bar{\psi}_{3}^{\varepsilon}}{\partial x_{3}} \\
& -\frac{1}{\varepsilon^{2}} \int_{\Omega_{M}} f \cdot \bar{\psi}^{\varepsilon} \quad \forall \psi^{\varepsilon} \in\left(H_{0}^{1}\left(\Omega_{\varepsilon}\right)\right)^{3} .
\end{aligned}
$$


We now take in (5.5) $\psi^{\varepsilon}\left(x, x_{3}\right)=\psi\left(x, \frac{x}{\varepsilon}, x_{3}\right)$ with $\psi \in \mathcal{V}^{0}$ and obtain

$$
\begin{aligned}
& \int_{\Omega_{M}} \bar{p}^{\varepsilon} \sum_{i=1}^{2} \frac{\partial \psi_{i}}{\partial x_{i}}=\frac{\mu}{2} \sum_{i, j=1}^{2} \int_{\Omega_{M}}\left[\frac{\partial \overline{u_{i}^{\varepsilon}}}{\partial x_{j}}+\frac{\partial \bar{u}_{j}^{\varepsilon}}{\partial x_{i}}\right]\left[\frac{\partial \psi_{i}}{\partial x_{j}}+\frac{1}{\varepsilon} \frac{\partial \psi_{i}}{\partial y_{j}}+\frac{\partial \psi_{j}}{\partial x_{i}}+\frac{1}{\varepsilon} \frac{\partial \psi_{j}}{\partial y_{i}}\right] \\
& +\mu \sum_{i=1}^{2} \int_{\Omega_{M}}\left[\frac{1}{\lambda \varepsilon} \frac{\partial \bar{u}_{i}^{\varepsilon}}{\partial x_{3}}+\frac{\partial \bar{u}_{3}^{\varepsilon}}{\partial x_{i}}\right]\left[\frac{1}{\lambda \varepsilon} \frac{\partial \psi_{i}}{\partial x_{3}}+\frac{\partial \psi_{3}}{\partial x_{i}}+\frac{1}{\varepsilon} \frac{\partial \psi_{3}}{\partial y_{i}}\right]+\frac{2 \mu}{\lambda^{2} \varepsilon^{2}} \int_{\Omega_{M}} \frac{\partial \bar{u}_{3}^{\varepsilon}}{\partial x_{3}} \frac{\partial \psi_{3}}{\partial x_{3}} \\
& -\frac{1}{\varepsilon^{2}} \int_{\Omega_{M}} f \cdot \bar{\psi}^{\varepsilon}
\end{aligned}
$$

Passing to the two-scale limit in $\Omega_{M}$, after multiplying by $\varepsilon^{2}$, and using Theorems 4.1 and 4.2, we deduce

$$
\begin{aligned}
& \int_{Q} p(x)\left(\sum_{i=1}^{2} \frac{\partial \psi_{i}}{\partial x_{i}}\right)=\frac{\mu}{2} \sum_{i, j=1}^{2} \int_{Q}\left(\frac{\partial u_{i}}{\partial y_{j}}+\frac{\partial u_{j}}{\partial y_{i}}\right)\left(\frac{\partial \psi_{i}}{\partial y_{j}}+\frac{\partial \psi_{j}}{\partial y_{i}}\right) \\
& +\mu \sum_{i=1}^{2} \int_{Q}\left(\frac{1}{\lambda} \frac{\partial u_{i}}{\partial x_{3}}+\frac{1}{\lambda} \frac{\partial v_{i}}{\partial y_{3}}+\frac{\partial u_{3}}{\partial y_{i}}\right)\left(\frac{1}{\lambda} \frac{\partial \psi_{i}}{\partial x_{3}}+\frac{\partial \psi_{3}}{\partial y_{i}}\right) \\
& +\frac{2 \mu}{\lambda^{2}} \int_{Q}\left(\frac{\partial u_{3}}{\partial x_{3}}+\frac{\partial v_{3}}{\partial y_{3}}\right) \frac{\partial \psi_{3}}{\partial x_{3}}-\int_{Q} f \cdot \psi \cdot
\end{aligned}
$$

As $\psi, u$ do not depend on $y_{3}$, and $v_{i}$ is $y_{3}$-periodic, we deduce

$$
\begin{aligned}
\int_{Q} p(x) \sum_{i=1}^{2} \frac{\partial \psi_{i}}{\partial x_{i}} d y d x d x_{3}=2 \mu \sum_{i, j=1}^{3} & \int_{Q} D_{i, j}^{B}(u) D_{i, j}^{B}(\psi) d y d x d x_{3} \\
& -\int_{Q} f \cdot \psi d y d x d x_{3} \quad \forall \psi \in \mathcal{V}^{0}
\end{aligned}
$$

Now we prove exactly as in [9], taking $\psi=(\theta(x) \bar{\xi}, 0,0)$ as a test function in (5.6) with $\theta$ arbitrary in $\mathcal{D}(\omega)$ and $\bar{\xi}$ the extension on $B_{x}$ of a function depending only on $y_{2}$ and $x_{3}$ defined on $[0,1] \times\left[0, h_{m}\right]$ with nonzero average, that $\frac{\partial p}{\partial x_{1}} \in L^{2}(\omega)$ and the same holds for $\frac{\partial p}{\partial x_{2}} \in L^{2}(\omega)$. Then $p$ is in $H^{1}(\omega)$.

Now taking in (5.6) $\psi\left(x, y, x_{3}\right)=\theta(x) \varphi\left(x, y, x_{3}\right)$ with $\theta$ in $\mathcal{D}(\omega)$ and $\varphi$ in $\mathcal{V}$ arbitrary, and using Green's formula in the left-hand side of (5.6), we obtain

$$
\begin{aligned}
-\int_{B_{x}} \sum_{i=1}^{2} \frac{\partial p}{\partial x_{i}} \varphi_{i} d y d x_{3}=-2 \mu & \sum_{i, j=1}^{3} \int_{B_{x}} D_{i, j}^{B}(u) D_{i, j}^{B}(\varphi) d y d x_{3} \\
& -\sum_{i=1}^{3} \int_{B_{x}} f_{i} \varphi_{i} d y d x_{3} \quad \text { a.e. } x \in \omega .
\end{aligned}
$$

(Remark that $D_{i, j}^{B} u(x, \cdot) \in L^{2}\left(B_{x}\right)$ a.e. $x \in \omega$ by Fubini's theorem.) Using Lemma 5.2 we deduce that (5.7) is true for any $\varphi \in \mathcal{V}_{x}$. Now using the density of $\mathcal{V}_{x}$ in $\mathrm{V}_{0, x}$ we have the expected result. 
6. The variational inequality between $p$ and $u$. In the following we propose to obtain another variational relation between $p$ and $u$, more general than in Theorem 5.3 . Now the test functions will not vanish on $x_{3}=0$ (except for the third component) and the equality becomes a variational inequality. We first need to introduce the following spaces: $\mathcal{C}_{0 \#}^{1}(\overline{\omega \times Y})=\left\{v \in \mathcal{C}^{1}(\overline{\omega \times Y})\right.$ with $v(x, \cdot)=0$ for $x \in \partial \omega$ and $v(x, \cdot) y$-periodic on $Y$ \}, which is a Banach space as a closed subspace of $\mathcal{C}^{1}(\overline{\omega \times Y})$.

We then introduce $\mathcal{U}=\left\{v \in\left(L^{2}(] 0, h_{M}\left[; \mathcal{C}_{0 \#}^{1}(\overline{\omega \times Y})\right)\right)^{3}\right.$ with $v=0$ for $x_{3} \geq h(x, y)$, $v_{3}=0$ for $x_{3}=0, \frac{\partial v}{\partial x_{3}} \in\left(L^{2}(] 0, h_{M}\left[; \mathcal{C}_{0 \#}^{1}(\overline{\omega \times Y})\right)\right)^{3}$, and $\left.\frac{\partial v_{1}}{\partial y_{1}}+\frac{\partial v_{2}}{\partial y_{2}}+\frac{1}{\lambda} \frac{\partial v_{3}}{\partial x_{3}}=0\right\}$.

We need also the set $\mathrm{U}=\left\{v \in\left(L^{2}\left(Q_{M}\right)\right)^{3}: v=0\right.$ for $x_{3} \geq h(x, y) ; v_{3}=0$ for $x_{3}=0, \frac{\partial v}{\partial y_{i}} \in\left(L^{2}\left(Q_{M}\right)\right)^{3}, \quad i=1,2 ; \frac{\partial v}{\partial x_{3}} \in\left(L^{2}\left(Q_{M}\right)\right)^{3} ; v$ is $y$-periodic, and $\frac{\partial v_{1}}{\partial y_{1}}+\frac{\partial v_{2}}{\partial y_{2}}+$ $\left.\frac{1}{\lambda} \frac{\partial v_{3}}{\partial x_{3}}=0\right\}$.

$\mathrm{U}$ is a Hilbert space endowed with the norm

$$
\|v\|_{\mathrm{U}}^{2}=\left\|\frac{\partial v}{\partial x_{3}}\right\|_{\left(L^{2}\left(Q_{M}\right)\right)^{3}}+\sum_{i=1}^{2}\left\|\frac{\partial v}{\partial y_{i}}\right\|_{\left(L^{2}\left(Q_{M}\right)\right)^{3}} .
$$

It is clear from Theorem 4.2 that $u \in \mathrm{U}$. We have the following density result.

Proposition 6.1. $\mathcal{U}$ is dense in $\mathrm{U}$ for the norm of $\mathrm{U}$.

Proof. We can suppose that 0 is in the interior of $\omega$. We set for any $\sigma>1, \sigma$ near 1 ,

$$
Q_{m \sigma}=\left\{\left(x, y, x_{3}\right): \quad \sigma x \in \omega ; \quad \sigma x_{3} \in\right] 0, h_{M}[; \quad y \in Y\} \subset Q_{M} .
$$

For any $v \in \mathrm{U}$ we set $v^{\sigma}=\left(v_{1}^{\sigma}, v_{2}^{\sigma}, v_{3}^{\sigma}\right)$ such that, for $k=1,2$,

$$
\begin{gathered}
v_{k}^{\sigma}\left(x, y, x_{3}\right)= \begin{cases}v_{k}\left(\sigma x, y, \sigma x_{3}\right) & \text { for }\left(x, y, x_{3}\right) \in Q_{m \sigma}, \\
0 & \text { for }\left(x, y, x_{3}\right) \in Q_{M}-Q_{m \sigma},\end{cases} \\
v_{3}^{\sigma}\left(x, y, x_{3}\right)= \begin{cases}\frac{1}{\sigma} v_{3}\left(\sigma x, y, \sigma x_{3}\right) & \text { for }\left(x, y, x_{3}\right) \in Q_{m \sigma}, \\
0 & \text { for }\left(x, y, x_{3}\right) \in Q_{M}-Q_{m \sigma} .\end{cases}
\end{gathered}
$$

It is clear that $v^{\sigma} \in \mathrm{U}$ and $v^{\sigma} \rightarrow v$ in $\mathrm{U}$ for $\sigma \rightarrow 1$. We extend $v^{\sigma}$ by $y$-periodicity on $\omega \times]-1,2\left[{ }^{2} \times\right] 0, h_{M}\left[\right.$. Then we extend it by 0 on $\left.\mathbb{R}^{4} \times\right] 0, h_{M}[$. We then introduce, for any $\delta>0$ small enough, the function $v^{\sigma \delta}=v^{\sigma} \star_{x, y} \rho_{\delta}$ (defined by convolution in $x, y$ only) where $\rho_{\delta} \in \mathcal{D}\left(\mathbb{R}^{4}\right)$ is a sequence of mollifier functions. We have

$$
\begin{aligned}
v^{\sigma \delta}\left(x, y, x_{3}\right) & =\int_{\mathbb{R}^{4}} v^{\sigma}\left(x-t, y-z, x_{3}\right) \rho_{\delta}(t, z) d t d z \\
& =\int_{\mathbb{R}^{4}} v^{\sigma}\left(t, z, x_{3}\right) \rho_{\delta}(x-t, y-z) d t d z .
\end{aligned}
$$

For $\sigma>1$ we have that $v^{\sigma \delta}$ is an element of $\mathcal{U}$ for $\delta$ small enough. (Remark that

$$
\left.\frac{\partial v^{\sigma \delta}}{\partial y_{k}}=\frac{\partial v^{\sigma}}{\partial y_{k}} \star_{x, y} \rho_{\delta} \quad \text { for } \quad k=1,2 \quad \text { and } \quad \frac{\partial v^{\sigma \delta}}{\partial x_{3}}=\frac{\partial v^{\sigma}}{\partial x_{3}} \star_{x, y} \rho_{\delta} .\right)
$$

We also prove classically that for fixed $\sigma>1$ we have $v^{\sigma \delta} \rightarrow v^{\sigma}$ in $\mathrm{U}$ for $\delta \rightarrow 0$. This ends the proof.

We can now obtain the following variational inequality. 
Theorem 6.1. The two-scale limits $p$ and $u$ satisfy the following relation:

$$
\begin{aligned}
& 2 \mu \sum_{i, j=1}^{3} \int_{Q} D_{i, j}^{B}(u) D_{i, j}^{B}(\varphi) d y d x d x_{3}+j_{0}(u+\varphi)-j_{0}(u) \\
& +\sum_{i=1}^{2} \int_{Q} \varphi_{i} \frac{\partial p}{\partial x_{i}} d y d x d x_{3} \geq \int_{Q} f \cdot \varphi d y d x d x_{3} \quad \forall \varphi \in \mathrm{U},
\end{aligned}
$$

where

$$
j_{0}(\psi)=\frac{1}{\lambda} \int_{\omega} \int_{Y} k(x)\left|\underline{\psi}(x, y, 0)-s^{0}\right| d y d x \quad \forall \psi \in \mathrm{U} .
$$

Proof. Taking $\varphi^{\varepsilon}$ as a test function in (3.2) such that

$$
\varphi^{\varepsilon}\left(x, x_{3}\right)=\left(G_{1}, G_{2}, \lambda \varepsilon G_{3}\right)\left(x, x_{3}\right)+\left(\psi_{1}, \psi_{2}, \psi_{3}\right)\left(x, \frac{x}{\varepsilon}, x_{3}\right)
$$

with $\psi \in \mathcal{U}$, and using the fact that $\operatorname{div}_{x, x_{3}} G=0$,

$$
\frac{\partial \psi_{1}}{\partial y_{1}}+\frac{\partial \psi_{2}}{\partial y_{2}}+\frac{1}{\lambda} \frac{\partial \psi_{3}}{\partial x_{3}}=0, \quad \text { and } \quad G_{i}(x, 0)=s_{i}^{0}
$$

we obtain

$$
\begin{aligned}
& \frac{\mu}{2} \sum_{i, j=1}^{2} \int_{\Omega_{M}}\left(\frac{\partial \overline{u_{i}^{\varepsilon}}}{\partial x_{j}}+\frac{\partial \overline{u_{j}^{\varepsilon}}}{\partial x_{i}}\right)\left(\frac{\partial \psi_{i}}{\partial x_{j}}+\frac{1}{\varepsilon} \frac{\partial \psi_{i}}{\partial y_{j}}+\frac{\partial \psi_{j}}{\partial x_{i}}+\frac{1}{\varepsilon} \frac{\partial \psi_{j}}{\partial y_{i}}\right) \\
& +\frac{\mu}{2} \sum_{i=1}^{2} \int_{\Omega_{M}}\left(\frac{\partial \overline{u_{i}^{\varepsilon}}}{\partial x_{j}}+\frac{\partial \overline{u_{j}^{\varepsilon}}}{\partial x_{i}}\right)\left(\frac{\partial G_{i}}{\partial x_{j}}+\frac{\partial G_{j}}{\partial x_{i}}\right) \\
& +\mu \sum_{i=1}^{2} \int_{\Omega_{M}}\left(\frac{1}{\lambda \varepsilon} \frac{\partial \overline{u_{i}^{\varepsilon}}}{\partial x_{3}}+\frac{\partial \overline{u_{3}^{\varepsilon}}}{\partial x_{i}}\right)\left(\frac{1}{\lambda \varepsilon} \frac{\partial \psi_{i}}{\partial x_{3}}+\frac{\partial \psi_{3}}{\partial x_{i}}+\frac{1}{\varepsilon} \frac{\partial \psi_{3}}{\partial y_{i}}\right) \\
& +\mu \sum_{i=1}^{2} \int_{\Omega_{M}}\left(\frac{1}{\lambda \varepsilon} \frac{\partial \overline{u_{i}^{\varepsilon}}}{\partial x_{3}}+\frac{\partial \overline{u_{3}^{\varepsilon}}}{\partial x_{i}}\right)\left(\frac{1}{\lambda \varepsilon} \frac{\partial G_{i}}{\partial x_{3}}+\lambda \varepsilon \frac{\partial G_{3}}{\partial x_{i}}\right)^{-\bar{\varepsilon}} \frac{\partial \psi_{3}}{\lambda^{2} \varepsilon^{2}} \int_{\Omega_{M}} \frac{\partial \mu}{\partial x_{3}} \frac{2 \mu}{\partial x_{3}} \int_{\Omega_{M}} \frac{\partial \bar{u}_{3}^{\varepsilon}}{\partial x_{3}} \frac{\partial G_{3}}{\partial x_{3}}-\int_{\Omega_{M}} p^{\varepsilon} \sum_{i=1}^{2} \frac{\partial \psi_{i}}{\partial x_{i}} \\
& +\frac{1}{\lambda \varepsilon^{2}} \int_{\omega} k(x)\left|\underline{\psi}\left(x, \frac{x}{\varepsilon}, 0\right)\right| \geq \frac{\mu}{2} \sum_{i=1}^{2} \int_{\Omega_{M}}\left(\frac{\partial \bar{u}_{i}^{\varepsilon}}{\partial x_{j}}+\frac{\partial \bar{u}_{j}^{\varepsilon}}{\partial x_{i}}\right)^{2}+\frac{2 \mu}{\lambda^{2} \varepsilon^{2}} \int_{\Omega_{M}}\left(\frac{\partial \bar{u}_{3}^{\varepsilon}}{\partial x_{3}}\right)^{2} \\
& +\mu \int_{\Omega_{M}}\left(\frac{1}{\lambda \varepsilon} \frac{\partial \bar{u}_{i}^{\varepsilon}}{\partial x_{3}}+\frac{\partial \bar{u}_{i}^{\varepsilon}}{\partial x_{i}}\right)^{2}+\frac{1}{\lambda \varepsilon^{2}} \int_{\omega} k(x)\left|\underline{u}^{\varepsilon}(x, 0)-s^{0}\right| d x \\
& +\frac{1}{\varepsilon^{2}} \int_{\Omega_{M}} f \cdot\left(\psi-\bar{u}^{\varepsilon}\right)+\frac{1}{\varepsilon^{2}} \int_{\Omega_{M}}^{f} \cdot\left(G_{1}, G_{2}, \lambda \varepsilon G_{3}\right)^{T} .
\end{aligned}
$$

We multiply by $\varepsilon^{2}$ and we pass to the limit in $\varepsilon$ using Theorems 4.1, 4.2 and the fact that $p \in H^{1}(\omega)$. From the lower semicontinuity result, Theorem 8.5 and the fact that the application $(x, z) \in \omega \times \mathbb{R}^{2} \mapsto k(x)\left|z-s^{0}\right| \in \mathbb{R}$ satisfies the hypotheses $(H 1)-(H 5)$ 
of Section 8, we obtain

$$
\liminf _{\varepsilon \rightarrow 0} \int_{\omega} k(x)\left|\underline{u}^{\varepsilon}(x, 0)-s^{0}\right| d x \geq \int_{\omega} \int_{Y} k(x)\left|\underline{u}(x, y, 0)-s^{0}\right| d y d x .
$$

We then have

$$
\begin{aligned}
2 \mu \sum_{i, j=1}^{3} \int_{Q} D_{i, j}^{B}(u) D_{i, j}^{B}(\psi) & +\frac{2 \mu}{\lambda} \sum_{i=1}^{2} \int_{Q} D_{i, 3}^{B}(u) \frac{\partial G_{i}}{\partial x_{3}}+\sum_{i=1}^{2} \int_{Q} \psi_{i} \frac{\partial p}{\partial x_{i}} \\
+\frac{1}{\lambda} \int_{\omega} \int_{Y} k|\underline{\psi}(x, y, 0)| \geq 2 \mu & \sum_{i, j=1}^{3} \int_{Q}\left(D_{i, j}^{B}(u)\right)^{2}+\frac{1}{\lambda} \int_{\omega} \int_{Y} k\left|\underline{u}(x, y, 0)-s^{0}\right| \\
& \left.+\int_{Q} f \cdot\left(\psi-u+\left(G_{1}, G_{2}, 0\right)^{T}\right)\right) \quad \forall \psi \in \mathcal{U} .
\end{aligned}
$$

By the density of $\mathcal{U}$ in $\mathrm{U}$ (Proposition 6.1) we deduce that the above variational inequality is satisfied for any $\psi$ in $\mathrm{U}$. Then we take in (6.4) $\psi=u+\varphi-\left(G_{1}, G_{2}, 0\right)^{T}$ with $\varphi$ an arbitrary element of $U$ and we obtain the desired result, provided that we prove

$$
\int_{Q} \sum_{i=1}^{2} u_{i} \frac{\partial p}{\partial x_{i}}=\int_{Q} \sum_{i=1}^{2} G_{i} \frac{\partial p}{\partial x_{i}} .
$$

From (4.9) we have

$$
\int_{Q} \sum_{i=1}^{2} u_{i} \frac{\partial p}{\partial x_{i}}=\int_{\partial \omega} p \sum_{i=1}^{2} \tilde{g}_{i} n_{i} .
$$

On the other hand, using Green's formula we can write

$$
\int_{Q} p \sum_{i=1}^{2} \frac{\partial G_{i}}{\partial x_{i}}=-\int_{Q} \sum_{i=1}^{2} G_{i} \frac{\partial p}{\partial x_{i}}+\int_{\partial \omega} \int_{Y} p \sum_{i=1}^{2} g_{i} n_{i} .
$$

Taking into account that $\operatorname{div}_{x, x_{3}} G=0$ we deduce

$$
\int_{Q} \sum_{i=1}^{2} G_{i} \frac{\partial p}{\partial x_{1}}=\int_{\partial \omega} \int_{Y} p \sum_{i=1}^{2} \tilde{g}_{i} n_{i},
$$

which gives (6.5).

REMARK 6.2. A direct method to obtain the inequality (6.1) would be to take, as a test function, $\varphi^{\varepsilon}=\psi\left(x, \frac{x}{\varepsilon}, x_{3}\right)+u^{\varepsilon}$ in (3.2) with arbitrary $\psi$. But if we do that we obtain in the left side of (6.2),

$$
\frac{1}{\lambda \varepsilon^{2}} \int_{\omega} k(x)\left|\underline{u}^{\varepsilon}(x, 0)+\underline{\psi}\left(x, \frac{x}{\varepsilon}, 0\right)-s^{0}\right| d x
$$

instead of

$$
\frac{1}{\lambda \varepsilon^{2}} \int_{\omega} k(x)\left|\underline{\psi}\left(x, \frac{x}{\varepsilon}, 0\right)\right| d x .
$$

So, using the two-scale limit, we need to obtain

$$
\begin{aligned}
& \lim _{\varepsilon \rightarrow 0} \int_{\omega} k(x)\left|\underline{u}^{\varepsilon}(x, 0)+\underline{\psi}\left(x, \frac{x}{\varepsilon}, 0\right)-s^{0}\right| d x \\
= & \int_{\omega} \int_{Y} k(x)\left|\underline{u}(x, y, 0)+\underline{\psi}(x, y, 0)-s^{0}\right| d x
\end{aligned}
$$


and a strong convergence result such as

$$
\left\|\underline{u}^{\varepsilon}(x, 0)-\underline{u}\left(x, \frac{x}{\varepsilon}, 0\right)\right\|_{L^{2}(\omega \times Y)} \rightarrow 0
$$

would be necessary. But such a result seems very difficult to prove since the function $y \mapsto u(x, y, 0)$ is not continuous. Then

$$
(x, y) \mapsto k(x)\left|\underline{u}(x, y, 0)+\underline{\psi}(x, y, 0)-s^{0}\right|
$$

is not a Carathéodory function.

7. The limit problem. We shall denote $s^{\star}(x, y)=u(x, y, 0)$, which is one of the unknowns of our limit problem. For any $x \in \omega$ we recall the following space:

$$
\mathrm{V}_{0, x}=\left\{\varphi \in\left(H_{0 \#}^{1}\left(B_{x}\right)\right)^{3} \quad \text { with } \quad \frac{\partial \varphi_{1}}{\partial y_{1}}+\frac{\partial \varphi_{2}}{\partial y_{2}}+\frac{1}{\lambda} \frac{\partial \varphi_{3}}{\partial x_{3}}=0\right\}
$$

and introduce

$$
\mathrm{V}_{1, x}=\left\{\varphi \in\left(H_{1 \#}^{1}\left(B_{x}\right)\right)^{3} \quad \text { with } \quad \frac{\partial \varphi_{1}}{\partial y_{1}}+\frac{\partial \varphi_{2}}{\partial y_{2}}+\frac{1}{\lambda} \frac{\partial \varphi_{3}}{\partial x_{3}}=0\right\} .
$$

We define the following local problems:

$\left(L_{k}\right)$ : Find $w^{k} \in \mathrm{V}_{0, x}$ (for $\left.k=1,2\right)$ such that

$$
2 \mu \sum_{i, j=1}^{3} \int_{B_{x}} D_{i j}^{B}\left(w^{k}\right) D_{i j}^{B}(\varphi) d y d x_{3}=-\int_{B_{x}} \varphi_{k} d y d x_{3} \quad \forall \varphi \in \mathrm{V}_{0, x} .
$$

$\left(L_{3}\right)$ : Find $w^{\star} \in \mathrm{V}_{0, x}$ such that

$$
2 \mu \sum_{i, j=1}^{3} \int_{B_{x}} D_{i j}^{B}\left(w^{3}\right) D_{i j}^{B}(\varphi) d y d x_{3}=\int_{B_{x}} f \cdot \varphi d y d x_{3} \quad \forall \varphi \in \mathrm{V}_{0, x} .
$$

On the other hand, we introduce the following space of traces on $x_{3}=0: S_{x}=$ $\left\{\theta \in\left(L^{2}(Y)\right)^{2}: \exists \varphi \in \mathrm{V}_{1, x}\right.$ such that $\left.\varphi_{k}(y, 0)=\theta_{k}(y), k=1,2, \varphi_{3}(y, 0)=0\right\}$ and $S=\left\{\tilde{\theta} \in\left(L^{2}(\omega \times Y)\right)^{2}: \quad \exists \tilde{\varphi} \in \mathrm{U}\right.$ such that $\left.\tilde{\varphi}_{k}(x, y, 0)=\tilde{\theta}_{k}(x, y), \quad k=1,2\right\}$.

It is clear that $s^{\star}(x, \cdot) \in S_{x}$ a.e. $x \in \omega$ and $s^{\star} \in S$. For any $s \in S$ we define the problem

$(L)$ : Find $w \in \mathrm{V}_{1, x}$ such that $w=(s(x, \cdot), 0)$ for $x_{3}=0$ and

$$
2 \mu \sum_{i, j=1}^{3} \int_{B_{x}} D_{i j}^{B}(w) D_{i j}^{B}(\varphi) d y d x_{3}=0 \quad \forall \varphi \in \mathrm{V}_{0, x} .
$$

It is clear from the Korn inequality that $\left(L_{k}\right)_{k=1,2,3}$ and $(L)$ are well-posed problems. We denote in the following by $\mathcal{R}$ the linear operator from $S$ to $\mathrm{U}$ such that $\mathcal{R}(s)=w$, where $w(x, \cdot)$ is the solution of the problem $(L)$ a.e. $x$ in $\omega$. We introduce the following scalar product on $S$ :

$$
(s, \tau)_{S}=(\mathcal{R}(s), \mathcal{R}(\tau))_{\mathrm{U}}
$$

We have

LEmma 7.1. $S$ endowed with the scalar product (7.4) is a Hilbert space. 
Proof. Since $\mathcal{R}$ is injective from $S$ to $\mathrm{U}$ (since $\operatorname{Ker}(\mathcal{R})=\{0\}$ ) it suffices to prove that $\mathcal{R}(s)$ is closed in $U$. Let $w^{k}=\mathcal{R}\left(s^{k}\right)$ be a sequence in $\mathcal{R}(S)$ such that $w^{k} \rightarrow w$ in $\mathrm{U}$. Then for any $\varphi \in \mathcal{V}$ we have (7.3) with $w$ replaced by $w^{k}$. Multiplying by an arbitrary $\theta \in \mathcal{D}(\omega)$, integrating in $\omega$ and passing to the limit in $k$ we obtain

$$
\sum_{i, j=1}^{3} \int_{\omega} \int_{B_{x}} D_{i j}^{B}(w) D_{i j}^{B}(\varphi) d y d x_{3} \theta(x) d x=0,
$$

which implies, since $\theta$ is arbitrary, that

$$
\sum_{i, j=1}^{3} \int_{B_{x}} D_{i j}^{B}(w) D_{i j}^{B}(\varphi) d y d x_{3}=0, \quad \text { a.e. } x \in \omega .
$$

Now set $s(x, y)=\underline{w}(x, y, 0)$, so $s \in S$. On the other hand, the equality (7.5) is true for any $\varphi \in \mathcal{V}$. Using Lemma 5.2 and the density of $\mathcal{V}_{x}$ in $V_{0, x}$ we deduce that $w=\mathcal{R}(s)$, which ends the proof.

REMARK 7.2. It is clear from Lemma 7.1 that $\mathcal{R} \in \mathcal{L}(S, \mathrm{U})$.

We have the following result whose proof is elementary.

Proposition 7.1. There exists a constant $C>0$ such that

$$
\left\|w^{k}\right\|_{\left(H^{1}\left(B_{x}\right)\right)^{3}} \leq C \quad \text { for } \quad k=1,2,3 \quad \text { a.e. } \quad x \in \omega,
$$

where $w^{k}$ is the solution of the problem $\left(L_{k}\right)$.

We now have

Proposition 7.2. The limit $u$ can be written in the following manner: a.e. $x \in \omega$ we have

$$
u=\sum_{k=1}^{2} w^{k} \frac{\partial p}{\partial x_{k}}+w^{3}+w^{\star} \quad \text { a.e. } \quad\left(y, x_{3}\right) \in B_{x}
$$

with $w^{k}$ solutions of the problem $\left(L_{k}\right)_{k=1,2,3}$ and $w^{\star}=\mathcal{R}\left(s^{\star}\right)$.

Moreover $p$ satisfies the following homogenized Reynolds equation:

$$
\int_{\omega} A \nabla p \cdot \nabla v=\int_{\omega} \sum_{j=1}^{2}\left[\int_{B_{x}}\left(w_{j}^{\star}+w_{j}^{3}\right) d y d x_{3}\right] \frac{\partial v}{\partial x_{j}} d x-\int_{\partial \omega} \tilde{g} \cdot \nu v \quad \forall v \in H^{1}(\omega),
$$

where $A$ is the matrix defined by

$$
A_{j, k}=-\int_{B_{x}} w_{j}^{k} d y d x_{3} \quad j=1,2, \quad k=1,2 .
$$

Proof. The relation (7.6) is easily obtained from (7.1)-(7.3), (5.4) by linearity and uniqueness and (7.7) is an immediate consequence of (7.6) and (4.9).

We shall need to consider the equality (7.7) with the supplementary condition $\int_{\omega} p d x=$ 0 (which is proved in Theorem 4.1) as a well-posed problem in $p$. Remark also that by hypothesis (2.6) we have $\int_{\partial \omega} \tilde{g} \cdot v=0$. This will be a classical consequence of the following result. 
Lemma 7.3. The matrix $A$ belongs to $\left(L^{\infty}(\omega)\right)^{4}$ and is symmetric and positive definite uniformly in $x$; that is, there exists a constant $\alpha>0$ such that

$$
\xi^{T} A \xi \geq \alpha\|\xi\|^{2} \quad \forall \xi \in \mathbb{R}^{2} .
$$

Proof. We proceed as in 9]. The fact that $A$ belongs to $\left(L^{\infty}(\omega)\right)^{4}$ is an immediate consequence of Proposition 7.1. Taking $w^{l}$ as a test function in $\left(L_{k}\right)(k=1,2)$, we obtain

$$
A_{k, l}=2 \mu \sum_{i, j=1}^{3} \int_{B_{x}} D_{i j}^{B}\left(w^{k}\right) D_{i j}^{B}\left(w^{l}\right),
$$

which gives the symmetry of $A$. We also deduce

$$
\sum_{k, l=1}^{2} A_{k, l} \xi_{k} \xi_{l}=2 \mu \sum_{i, j=1}^{2} \int_{B_{x}}\left|D_{i j}^{B}(\tilde{w})\right|^{2} \quad \text { with } \tilde{w}=\xi_{1} w_{1}+\xi_{2} w_{2} .
$$

On the other hand, multiplying $\left(L_{k}\right)$ by $\xi_{k}$ and adding in $k$ we get

$$
2 \mu \sum_{i, j=1}^{3} \int_{B_{x}} D_{i j}^{B}(\tilde{w}) D_{i j}^{B}(\varphi)=-\int_{B_{x}}\left(\xi_{1} \varphi_{1}+\xi_{2} \varphi_{2}\right) \quad \forall \varphi \in \mathrm{V}_{0, x} .
$$

For any fixed $\xi$ we choose $\varphi$ in the following manner:

$$
\varphi\left(y, x_{3}\right)=\left(-\xi_{1} x_{3}\left(h_{m}-x_{3}\right),-\xi_{2} x_{3}\left(h_{m}-x_{3}\right), 0\right)
$$

for $x_{3} \in\left[0, h_{m}\right]$ and extended by 0 on $B_{x}$. It is clear that $\varphi \in \mathrm{V}_{0, x}$ for any $x \in \omega$; then it can be taken as a test function in (7.10). We obtain, using also the Cauchy-Schwarz inequality,

$$
\frac{h_{m}^{3}}{6}\|\xi\|^{2} \leq 2 \mu \sqrt{\sum_{i, j=1}^{3} \int_{B_{x}}\left|D_{i j}^{B}(\tilde{w})\right|^{2}} \sqrt{\sum_{i, j=1}^{3} \int_{B_{x}}\left|D_{i j}^{B}(\varphi)\right|^{2}} .
$$

With the help of (7.9) we easily obtain (7.8).

For any $s \in S$ let us define $q$ as the unique solution of the problem

$\left(P_{0}\right)$ : Find $q \in H^{1}(\omega) / \mathbb{R}$ such that

$$
\int_{\omega} A \nabla q \nabla \varphi=\int_{\omega} \sum_{j=1}^{2}\left(\int_{B_{x}} w_{j} d y d x_{3}\right) \frac{\partial \varphi}{\partial x_{j}} \quad \forall \varphi \in H^{1}(\omega)
$$

with $w=\mathcal{R}(s)$ (the solution of (L)).

We denote by $\mathcal{Q}$ the linear operator from $\mathrm{U}$ to $H^{1}(\omega)$ such that $\mathcal{Q}(w)=q$ is a solution of $\left(P_{0}\right)$ for any $w \in \mathrm{U}$. It is obvious that $\mathcal{Q}$ is also continuous, that is,

$$
\mathcal{Q} \in \mathcal{L}\left(\mathrm{U}, H^{1}(\omega)\right) .
$$

On the other hand, we define $p_{1}$ as the unique solution of the problem

$\left(P_{1}\right)$ : Find $p_{1} \in H^{1}(\omega) / \mathbb{R}$ such that

$$
\int_{\omega} A \nabla p_{1} \cdot \nabla \varphi=\int_{\omega} \sum_{j=1}^{2}\left(\int_{B_{x}} w_{j}^{3} d y d x_{3}\right) \frac{\partial \varphi}{\partial x_{j}}-\int_{\partial \omega} \tilde{g} \cdot \nu \varphi \quad \forall \varphi \in H^{1}(\omega) / \mathbb{R} .
$$


By linearity and uniqueness we easily see that $p$ can be expressed as a function of $s^{\star}$ in the following manner:

$$
p=p_{0}+p_{1}
$$

with $p_{0}=\mathcal{Q} \circ \mathcal{R}\left(s^{\star}\right)$ and $p_{1}$ the solution of $\left(P_{1}\right)$.

It is clear from (7.6) that $u$ can also be expressed as a function of $s^{\star}$ :

$$
u=\sum_{k=1}^{2} w^{k} \frac{\partial p_{0}}{\partial x_{k}}+w^{\star}+\sum_{k=1}^{2} w^{k} \frac{\partial p_{1}}{\partial x_{k}}+w^{3} .
$$

Now substituting (7.14) and (7.15) into (6.1) we obtain that $s^{\star}$ satisfies the following variational inequality:

$$
\begin{array}{r}
2 \mu \int_{Q} \sum_{i, j=1}^{3}\left[\sum_{k=1}^{2} D_{i j}^{B}\left(w^{k}\right) \frac{\partial p_{0}}{\partial x_{k}}+D_{i j}^{B}\left(w^{\star}\right)\right] \cdot D_{i j}^{B}(\varphi)+\int_{Q} \sum_{j=1}^{2} \frac{\partial p_{0}}{\partial x_{j}} \varphi_{j} \\
+2 \mu \int_{Q} \sum_{i, j=1}^{3}\left[\sum_{k=1}^{2} D_{i j}^{B}\left(w^{k}\right) \frac{\partial p_{1}}{\partial x_{k}}+D_{i j}^{B}\left(w^{3}\right)\right] \cdot D_{i j}^{B}(\varphi)+\int_{Q} \sum_{j=1}^{2} \frac{\partial p_{1}}{\partial x_{j}} \varphi_{j} \\
+\frac{1}{\lambda} \int_{\omega} \int_{Y} k(x)\left[\left|\underline{\varphi}(x, y, 0)+s^{\star}(x, y)-s^{0}\right|-\left|s^{\star}(x, y)-s^{0}\right|\right] \\
-\int_{Q} f \cdot \varphi \geq 0 \quad \forall \varphi \in \mathrm{U} .
\end{array}
$$

The first two integrals in the above inequality will be seen as a bilinear form while the three subsequent terms as a linear form. It is then natural to introduce the following bilinear form $b$ and linear form $T$ respectively:

$$
\begin{array}{r}
b(s, \theta)=2 \mu \int_{Q} \sum_{i, j=1}^{3}\left[\sum_{k=1}^{2} D_{i j}^{B}\left(w^{k}\right) \frac{\partial q}{\partial x_{k}}+D_{i j}^{B}(w)\right] \cdot D_{i j}^{B}(\psi) \\
+\int_{Q} \sum_{j=1}^{2} \frac{\partial q}{\partial x_{j}} \psi_{j}, \quad \forall(s, \theta) \in S \times S, \\
T(\theta)=2 \mu \int_{Q} \sum_{i, j=1}^{3}\left[\sum_{k=1}^{2} D_{i j}^{B}\left(w^{k}\right) \frac{\partial p_{1}}{\partial x_{k}}+D_{i j}^{B}\left(w^{3}\right)\right] \cdot D_{i j}^{B}(\psi) \\
+\int_{Q} \sum_{j=1}^{2} \frac{\partial p_{1}}{\partial x_{j}} \psi_{j}-\int_{Q} f \cdot \psi \quad \forall \theta \in S
\end{array}
$$

with $w=\mathcal{R}(s), q=\mathcal{Q}(\mathcal{R}(s))$, and $\psi$ an arbitrary relevement of $\theta$; that is, $\psi \in \mathrm{U}$ is such that $\psi_{\left.\right|_{x_{3}=0}}=\theta$.

Now we easily see that $b$ and $T$ are well-defined; that is, they are independent of the choice of the relevement $\psi$ of $\theta$. Indeed if $\psi^{1}$ and $\psi^{2}$ are two different relevements of $\theta$, then $\psi^{1}-\psi^{2}$ is an element of $\mathrm{V}_{0, x}$. Moreover the expressions of the right side and of (7.16) and (7.17) vanish for $\psi \in \mathrm{V}_{0, x}$ since $w^{k}$ satisfies $\left(L_{k}\right)$ for $k=1,2,3$. This proves that $b$ and $T$ are well defined. With the above notation the variational inequality (7.16) 
can be written in the following form:

$$
\text { Find } s^{\star} \in S: \quad b\left(s^{\star}, \theta\right)+T(\theta)+\hat{j}\left(s^{\star}+\theta\right)-\hat{j}\left(s^{\star}\right) \geq 0 \quad \forall \theta \in S
$$

where

$$
\hat{j}(\theta)=\frac{1}{\lambda} \int_{\omega} \int_{Y} k(x)\left|\theta(x, y)-s^{0}\right| d y d x .
$$

The expression of $b$ and $T$ can be simplified in the following manner.

Proposition 7.3. We have

$$
\begin{gathered}
b(s, \theta)=2 \mu \int_{Q} \sum_{i, j=1}^{3} D_{i j}^{B}(w) D_{i j}^{B}(\psi)+\int_{\omega} A \nabla q \cdot \nabla r \\
T(\theta)=2 \mu \int_{Q} \sum_{i, j=1}^{3} D_{i j}^{B}\left(w^{3}\right) D_{i j}^{B}(\psi)+\int_{Q} \sum_{j=1}^{2} \frac{\partial p_{1}}{\partial x_{j}} \psi_{j}-\int_{Q} f \cdot \psi
\end{gathered}
$$

with $w=\mathcal{R}(s), \psi=\mathcal{R}(\theta), q=\mathcal{Q}(\mathcal{R}(s))$ and $r=\mathcal{Q}(\mathcal{R}(\theta))$.

Proof. We take in (7.17) and (7.18) the relevement $\psi=\mathcal{R}(\theta)$. We then have

$$
\sum_{i, j=1}^{3} \int_{Q} D_{i j}^{B}\left(w^{k}\right) D_{i j}^{B}(\psi)=0, \quad k=1,2
$$

since $w^{k} \in \mathrm{V}_{0, x}$ and $\psi$ is the solution of (L) with $s$ replaced by $\theta$. On the other hand, considering $q=\mathcal{Q}(\mathcal{R}(s))$ as a test function in the problem $\left(P_{0}\right)$ satisfied by $r=\mathcal{Q}(\mathcal{R}(\theta))$ we find

$$
\int_{Q} \sum_{j=1}^{2} \frac{\partial q}{\partial x_{j}} \psi_{j}=\int_{\omega} \sum_{j=1}^{2}\left(\int_{B_{x}} \psi_{j}\right) \frac{\partial q}{\partial x_{j}}=\int_{\omega} A \nabla r \cdot \nabla q .
$$

This ends the proof.

We can now state the main result of this paper.

Theorem 7.4. The limit problem (7.19) satisfied by $s^{\star}=u(x, y, 0)$ has a unique solution $s^{\star} \in S$. Moreover for the entire sequence of $\varepsilon$ we have in $\Omega_{M}$,

$$
\begin{gathered}
\varepsilon^{2} \bar{p}^{\varepsilon} \rightarrow p_{0}+p_{1}, \\
\overline{u^{\varepsilon}} \rightarrow \rightarrow \sum_{k=1}^{2} w^{k} \frac{\partial p_{0}}{\partial x_{k}}+w^{\star}+\sum_{k=1}^{2} w^{k} \frac{\partial p_{1}}{\partial x_{k}}+w^{3},
\end{gathered}
$$

where $w^{k}$, for $k=1,2,3$, are solutions of $\left(L_{k}\right), p_{1}$ is a solution of $\left(P_{1}\right), w^{\star}=\mathcal{R}\left(s^{\star}\right)$, $p_{0}=\mathcal{Q}\left(\mathcal{R}\left(s^{\star}\right)\right)$. All the limit functions are defined on $Q$ and extended by 0 to $Q_{M}$.

Proof. We use the form of $b$ and $T$ given in Proposition 7.3. The symmetry of $b$ is obvious and the continuity of $b$ and $T$ are immediate from the fact that $A \in\left(L^{\infty}(\omega)\right)^{4}$ (Proposition 7.1) and that $\mathcal{R}$ and $\mathcal{Q}$ are linear and continuous (Remark 7.2) and (7.12). Moreover using positive definiteness of $A$ we get

$$
b(s, s) \geq \int_{Q} \sum_{i, j}^{3}\left|D_{i j}^{B}(w)\right|^{2} .
$$


By Korn's inequality and since $\|w\|_{\mathrm{U}}=\|s\|_{S}$ we deduce that there exists a constant $\alpha_{1}>0$ such that

$$
b(s, s) \geq \alpha_{1}\|s\|_{S}^{2} .
$$

Since $\hat{j}$ is convex and continuous in $S$ (we have the continuous embedding of $S$ in $L^{2}(\omega \times Y)$ ), and $S$ is a Hilbert space we deduce classically the existence and uniqueness of a solution of the variational inequality (7.19) (Proposition 2.2 chap. II of [17]). We deduce from the uniqueness that the entire sequences (in $\varepsilon$ ) $\bar{u}^{\varepsilon}$ and $\varepsilon^{2} \bar{p}^{\varepsilon}$ converge to the indicated limits.

8. Lower-semicontinuity result for the two-scale convergence. The aim of this chapter is to prove (6.3), in a generalized case, in Theorem 8.5, which is still an open problem, using some results on the subdifferential and regularization of convex functions.

Let $\Omega \subset \mathbb{R}^{m}$ be an open bounded domain, $Y=[0,1]^{m}$, and $\varphi: \Omega \times \mathbb{R}^{m} \times \mathbb{R}^{n} \rightarrow \mathbb{R}$ such that the following hypotheses hold:

(H1) $\varphi(x, \cdot, \cdot)$ is continuous a.e. $x \in \Omega$,

(H2) $\varphi(\cdot, y, z)$ is measurable for all $(y, z) \in \mathbb{R}^{m} \times \mathbb{R}^{n}$,

(H3) $\varphi(x, \cdot, z)$ is 1-periodic in $y$,

(H4) $\varphi(x, y, \cdot)$ is convex in $z$,

(H5) $\exists C_{1}>0$ and $\exists C_{2} \in L^{2}(\Omega)$ such that

$$
|\varphi(x, y, z)| \leq C_{1}\|z\|^{2}+C_{2}(x) \quad \text { a.e. } \quad x \in \Omega \quad \forall(y, z) \in \mathbb{R}^{m} \times \mathbb{R}^{n} .
$$

Remark 8.1. A particular case of Theorem 8.5, where $\varphi$ depends only on $z$, was proved in 18 and 19 (see also 20 for an analogous result for the stochastic two-scale convergence). All these results are valid only in the particular case where $\varphi$ is an affine function. Another particular case, with $\varphi(z)=z^{2}$, can be found in [15]. In [16] the case $\varphi(z)=|z|^{p}$ for $\left.p \in\right] 1,+\infty[$ was also considered, which is a particular case of our only for $p \in] 1,2]$.

For $\delta>0$ we define the partial Yosida-Moreau approximation of $\varphi$ :

$$
\varphi_{\delta}(x, y, z)=\inf _{s \in \mathbb{R}^{n}}\left\{\varphi(x, y, s)+\frac{\|z-s\|^{2}}{2 \delta}\right\},
$$

which is well defined (see for example [21, chapter 2, page 121, theorem 2.3) and for $(x, y) \in \Omega \times \mathbb{R}^{m}$ we consider the multivalued operator $A_{x, y}$ from $\mathbb{R}^{n}$ to $2^{\mathbb{R}^{n}}$ such that $A_{x, y}(z)=\partial_{z} \varphi(x, y, z)$, where $\partial_{z} \varphi$ is the subdifferential, in $z$, of $\varphi$ defined by

$$
z \mapsto \partial_{z} \varphi(x, y, z)=\left\{w \in \mathbb{R}^{n}: \quad \varphi(x, y, s) \geq \varphi(x, y, z)+w \cdot(s-z) \quad \forall s \in \mathbb{R}^{n}\right\} .
$$

From $(H 1)$ and $(H 4) \varphi$ is continuous and convex in $z$, so we have (see for example [17, page 22, Proposition 5.2) that

$$
\partial_{z} \varphi(x, y, z) \neq \emptyset ; \quad \text { then } \operatorname{Dom}\left(A_{x, y}\right)=\mathbb{R}^{n} .
$$

We have also (see for example 22], page 25, 21, theorem 2.1) that $A_{x, y}$ is a maximal monotone operator. Then (see for example [22, Proposition 2.2) we get

$$
\Im\left(I+\delta A_{x, y}\right)=\mathbb{R}^{n},
$$


so for all $\delta>0$, and for all $s \in \mathbb{R}^{n}$, there exists a unique $z \in \mathbb{R}^{n}$, such that

$$
\left(I+\delta A_{x, y}\right)(z) \ni s .
$$

We denote $z=\left(I+\delta A_{x, y}\right)^{-1}(s)$ and introduce the following application:

$$
J_{x, y, \delta}: \mathbb{R}^{n} \rightarrow \mathbb{R}^{n} \quad \text { such that } J_{x, y, \delta}(s)=\left(I+\delta A_{x, y}\right)^{-1}(s)
$$

and the partial Yosida approximation of the operator $A_{x, y}$,

$$
A_{x, y, \delta}: \mathbb{R}^{n} \rightarrow \mathbb{R}^{n} \quad \text { such that } A_{x, y, \delta}(s)=\frac{1}{\delta}\left(s-J_{x, y, \delta}(s)\right) .
$$

LEMMA 8.2. We have

$$
\begin{gathered}
\varphi_{\delta}(x, y, \cdot) \in \mathcal{C}^{1}\left(\mathbb{R}^{n}\right) \text { and is convex in } z, \\
\lim _{\delta \rightarrow 0} \varphi_{\delta}(x, y, z)=\varphi(x, y, z), \\
\nabla_{z} \varphi_{\delta}=A_{x, y, \delta}, \\
\lim _{\delta \rightarrow 0} J_{x, y, \delta}(z)=z \quad \forall z \in \mathbb{R}^{n}, \\
\left\|J_{x, y, \delta}\left(z_{1}\right)-J_{x, y, \delta}\left(z_{2}\right)\right\| \leq\left\|z_{1}-z_{2}\right\| \quad \forall\left(z_{1}, z_{2}\right) \in \mathbb{R}^{n} \times \mathbb{R}^{n}, \\
\left\|A_{x, y, \delta}\left(z_{1}\right)-A_{x, y, \delta}\left(z_{2}\right)\right\| \leq \frac{1}{\delta}\left\|z_{1}-z_{2}\right\| \quad \forall\left(z_{1}, z_{2}\right) \in \mathbb{R}^{n} \times \mathbb{R}^{n}, \\
\varphi\left(x, y, J_{x, y, \delta}(z)\right) \leq \varphi_{\delta}(x, y, z) \leq \varphi(x, y, z) \quad \forall z \in \mathbb{R}^{n} .
\end{gathered}
$$

Proof. The proof is similar to the well-known results in 21, page 121; see also [22].

We have the following technical result.

Proposition 8.1. For any $\xi$ such that $\xi(x, y) \in \partial_{z} \varphi(x, y, 0)$ we have

$$
\begin{gathered}
\nabla_{z} \varphi_{\delta}(x, y, \delta \xi(x, y))=\xi(x, y) \quad \text { a.e. } \quad x \in \omega \quad \forall y \in Y, \\
\left|\nabla_{z} \varphi_{\delta}(x, y, \delta \xi(x, y))\right| \leq \sqrt{n}\left(C_{1}+2 C_{2}(x)\right) .
\end{gathered}
$$

Proof. Since $\delta \xi \in \delta A_{x, y}(0)+0$ we deduce $J_{x, y, \delta}(\delta \xi)=0$, which implies

$$
\nabla_{z} \varphi_{\delta}(x, y, \delta \xi(x, y))=A_{x, y, \delta}(\delta \xi)=\xi(x, y),
$$

which proves (8.11). On the other hand, we have

$$
\varphi(x, y, z)-\varphi(x, y, 0) \geq \xi \cdot z \quad \forall z \in \mathbb{R}^{n} .
$$

Taking successively $z= \pm e_{k}$ in the above inequality where $e_{k}$ is the $k$-th element in the canonical basis in $\mathbb{R}^{n}$, we deduce using also $(H 5)$,

$$
\left|\xi_{k}\right| \leq C_{1}+2 C_{2}(x) \quad k=1, \ldots, n .
$$

With the help of (8.11) we obtain (8.12).

Proposition 8.2. We have

$$
\left|\varphi_{\delta}(x, y, z)\right| \leq 2 C_{1}\|z\|^{2}+C_{2}(x) \quad \text { a.e. } \quad x \in \Omega, \quad \forall(y, z) \in \mathbb{R}^{m} \times \mathbb{R}^{n},
$$

for all $0<\delta<\left(4 C_{1}\right)^{-1}$, and also

$$
\left|\nabla_{z} \varphi_{\delta}(x, y, z)\right| \leq 2 \sqrt{n}\left(C_{1}+2 C_{2}(x)\right)+\frac{\|z\|}{\delta} .
$$


Proof. From (8.10), $\varphi_{\delta}(x, y, z) \leq \varphi(x, y, z)$; then with $(H 5)$,

$$
\varphi_{\delta}(x, y, z) \leq 2 C_{1}\|z\|^{2}+C_{2}(x) .
$$

On the other hand, from the definition of $\varphi_{\delta}$ and $(H 5)$ we deduce

$$
\varphi_{\delta}(x, y, z) \geq-C_{2}(x)+\inf _{s \in \mathbb{R}^{n}}\left\{\frac{\|z-s\|^{2}}{2 \delta}-C_{1}\|s\|^{2}\right\} .
$$

Denoting $B(s)=\frac{\|z-s\|^{2}}{2 \delta}-C_{1}\|s\|^{2}$ we have

$$
B(s) \geq B\left(s_{\min }\right) \quad \forall s \in \mathbb{R}^{n} \quad \text { with } \quad s_{\min }=\frac{z}{1-2 \delta C_{1}}
$$

and we also have

$$
B\left(s_{\text {min }}\right)=-\frac{C_{1}}{1-2 \delta C_{1}}\|z\|^{2} .
$$

As $\delta<\left(4 C_{1}\right)^{-1}$, then $\frac{C_{1}}{1-2 \delta C_{1}} \leq 2 C_{1}$, so

$$
\varphi_{\delta}(x, y, z) \geq-2 C_{1}\|z\|^{2}-C_{2}(x) .
$$

Thus (8.13) follows. To prove (8.14), we write

$$
\nabla_{z} \varphi_{\delta}(x, y, z)=\nabla_{z} \varphi_{\delta}(x, y, \delta \xi(x, y))+\nabla_{z} \varphi_{\delta}(x, y, z)-\nabla_{z} \varphi_{\delta}(x, y, \delta \xi(x, y)),
$$

so from (8.9), (8.11) and (8.12) we deduce (8.14).

We introduce now the following operators:

$$
\begin{aligned}
S_{0}: L^{2}(\Omega \times Y) \rightarrow \mathbb{R}: & S_{0}(v)=\int_{\Omega} \int_{Y} \varphi(x, y, v(x, y)) d y d x, \\
S_{0}^{\delta}: L^{2}(\Omega \times Y) \rightarrow \mathbb{R}: & S_{0}^{\delta}(v)=\int_{\Omega} \int_{Y} \varphi_{\delta}(x, y, v(x, y)) d y d x,
\end{aligned}
$$

which are well defined from $(H 5)$ and (8.13). Recall the definition of the epigraph of an operator $S$ from $X$ to $\mathbb{R}, \operatorname{epi}(S):=\{(v, a) \in X \times \mathbb{R}: \quad a \geq S(v)\}$.

Proposition 8.3.

$$
\operatorname{epi}\left(S_{0}\right)=\bigcap_{\delta>0} \operatorname{epi}\left(S_{0}^{\delta}\right)
$$

Proof. From Lemma 8.2 (8.10) we have $S_{0}^{\delta}(v) \leq S_{0}(v)$ for all $v \in\left(L^{2}(\Omega)\right)^{n}$, so

$$
\operatorname{epi}\left(S_{0}\right) \subseteq \bigcap_{\delta>0} \operatorname{epi}\left(S_{0}^{\delta}\right) .
$$

Let $(v, a) \in \operatorname{epi}\left(S_{0}^{\delta}\right)$ for all $\delta>0$. Then $a \geq S_{0}^{\delta}(v)$. So taking $\delta \rightarrow 0$ using (8.5) and (8.13), we get the result by Lebesgue's dominated theorem.

We define now for all $v_{0} \in\left(L^{2}(\Omega \times Y)\right)^{n}$ and $\delta>0$

$$
\begin{gathered}
D_{v_{0}, \delta}=\left\{(v, a) \in\left(L^{2}(\Omega \times Y)\right)^{n} \times \mathbb{R}:\right. \\
\left.a \geq \int_{\Omega} \int_{Y}\left[\varphi_{\delta}\left(x, y, v_{0}(x, y)\right)+\nabla_{z} \varphi_{\delta}\left(x, y, v_{0}(x, y)\right)\left\{v(x, y)-v_{0}(x, y)\right\}\right] d y d x\right\} .
\end{gathered}
$$

The second integral is well defined from (8.13) and (8.14). 
LEMMA 8.3. For any $\delta>0$ we have

$$
\operatorname{epi}\left(S_{0}^{\delta}\right)=\bigcap_{v_{0} \in(\mathcal{D}(\Omega \times Y))^{n}} D_{v_{0}, \delta},
$$

where $(\mathcal{D}(\Omega \times Y))^{n}$ is the space of functions in $\left(\mathcal{C}^{\infty}(\Omega \times Y)\right)^{n}$ with compact supports in $\Omega \times Y$.

Proof. As $\varphi_{\delta}$ is a convex function in $z$ we have

$$
\begin{aligned}
\varphi_{\delta}(x, y, v(x, y)) & \geq \varphi_{\delta}\left(x, y, v_{0}(x, y)\right)+\nabla_{z} \varphi_{\delta}\left(x, y, v_{0}(x, y)\right) \cdot\left[(v(x, y))-\left(v_{0}(x, y)\right)\right] \\
& \forall v \in\left(L^{2}(\Omega \times Y)\right)^{n}, \quad \forall v_{0} \in(\mathcal{D}(\Omega \times Y))^{n}, \quad \forall \delta>0 .
\end{aligned}
$$

So by integration we easily obtain

$$
\begin{aligned}
a \geq & \int_{\Omega} \int_{Y} \varphi_{\delta}\left(x, y, v_{0}(x, y)\right) \\
& +\int_{\Omega} \int_{Y} \nabla_{z} \varphi_{\delta}\left(x, y, v_{0}(x, y)\right) \cdot\left\{v(x, y)-v_{0}(x, y)\right\} d y d x
\end{aligned}
$$

thus we deduce the first inclusion.

Let $(v, a) \in\left(L^{2}(\Omega \times Y)\right)^{n} \times \mathbb{R}$ satisfying (8.15) for all $v_{0} \in(\mathcal{D}(\Omega \times Y))^{n}$ and for a fixed $\delta>0$. By density there exists a sequence $\left(v_{k}\right) \in(\mathcal{D}(\Omega \times Y))^{n}$ such that $v_{k} \rightarrow v \in$ $\left(L^{2}(\Omega \times Y)\right)^{n}$. Taking $v_{0}=v_{k}$ in (8.15) we obtain

$$
\begin{aligned}
a \geq & \int_{\Omega} \int_{Y} \varphi_{\delta}\left(x, y, v_{k}(x, y)\right) d y d x \\
& +\int_{\Omega} \int_{Y} \nabla_{z} \varphi_{\delta}\left(x, y, v_{k}(x, y)\right) \cdot\left\{v(x, y)-v_{k}(x, y)\right\} d y d x .
\end{aligned}
$$

We now pass to the limit $k \rightarrow+\infty$ in the above inequality. For the first integral we have

$$
\begin{aligned}
& \int_{\Omega} \int_{Y}\left|\varphi_{\delta}\left(x, y, v_{k}\right)-\varphi_{\delta}(x, y, v)\right| d y d x \\
& \quad \leq \int_{\Omega} \int_{Y}\left|\nabla_{z} \varphi_{\delta}\left(x, y, v+\theta\left(v_{k}-v\right)\right)\right| \cdot\left|v_{k}-v\right| d y d x,
\end{aligned}
$$

with $0<\theta<1$. Then using (8.14) we have

$$
\int_{\Omega} \int_{Y}\left|\varphi_{\delta}\left(x, y, v_{k}\right)-\varphi_{\delta}(x, y, v)\right| d y d x \rightarrow 0 \quad \text { as } \quad k \rightarrow+\infty .
$$

Using also (8.14),$\left|\nabla_{z} \varphi_{\delta}\left(x, y, v_{k}(x, y)\right)\right|$ is bounded in $L^{2}(\Omega \times Y)$, so the second integral of (8.16) tends to zero. Thus we get

$$
a \geq \int_{\Omega} \int_{Y} \varphi_{\delta}(x, y, v(x, y)) d y d x
$$

so $(v, a) \in \operatorname{epi}\left(S_{0}^{\delta}\right)$.

Lemma 8.4. For any $\delta$ such that $0<\delta<\frac{1}{4 C_{1}}$, we have

i): The functions $y \mapsto \varphi_{\delta}(x, y, z)$ and $y \mapsto \nabla_{z} \varphi_{\delta}(x, y, z)$ are 1-periodic a.e. $x \in \Omega$, for all $z \in \mathbb{R}^{n}$. 
ii): The functions $(y, z) \mapsto \varphi_{\delta}(x, y, z)$ and $(y, z) \mapsto \nabla_{z} \varphi_{\delta}(x, y, z)$ are continuous a.e. $x \in \Omega$.

iii): The functions, from $\Omega$ to $\mathbb{R}: \quad x \mapsto \varphi_{\delta}(x, y, z)$ and $x \mapsto \nabla_{z} \varphi_{\delta}(x, y, z)$ are measurable for all $(y, z) \in Y \times \mathbb{R}^{n}$.

iv): For any compact $K \subset \mathbb{R}^{n}$ we have that

$$
x \mapsto \sup _{(y, z) \in Y \times K} \varphi_{\delta}(x, y, z) \quad \text { and } \quad x \mapsto \sup _{(y, z) \in Y \times K} \nabla_{z} \varphi_{\delta}(x, y, z)
$$

have a finite $L^{2}(\Omega)$-norm.

Proof. We recall that (see for example [22, p.25)

$$
\varphi_{\delta}(x, y, z)=\inf _{s \in \mathbb{R}^{n}}\left\{\varphi(x, y, s)+\frac{\|z-s\|^{2}}{2 \delta}\right\}=\varphi(x, y, \tilde{s})+\frac{\|z-\tilde{s}\|^{2}}{2 \delta},
$$

where $\tilde{s}=J_{x, y, \delta}(z)$. From the $y$-periodicity of $\varphi$ we easily deduce the $y$-periodicity of $\varphi_{\delta}$. Since from (8.6) and (8.3) we have

$$
\nabla_{z} \varphi_{\delta}(x, y, z)=\frac{1}{\delta}\left(z-J_{x, y, \delta}(z)\right)
$$

we deduce from (8.18) the $y$-periodicity of $\nabla_{z} \varphi_{\delta}$.

Let us fix $y^{0} \in \mathbb{R}^{m}, z^{0} \in \mathbb{R}^{n}$ and let $y_{k} \rightarrow y^{0}$ and $z_{k} \rightarrow z^{0}$. We set

$$
\left.s_{k}=J_{x, y_{k}, \delta}\left(z_{k}\right)\right), \quad s^{0}=J_{x, y^{0}, \delta}\left(z^{0}\right)
$$

and we have

$$
\begin{gathered}
\varphi_{\delta}\left(x, y_{k}, z_{k}\right)=\inf _{s \in \mathbb{R}^{n}}\left\{\varphi\left(x, y_{k}, s\right)+\frac{\left\|z_{k}-s\right\|^{2}}{2 \delta}\right\}=\varphi\left(x, y_{k}, s_{k}\right)+\frac{\left\|z_{k}-s_{k}\right\|^{2}}{2 \delta}, \\
\varphi_{\delta}\left(x, y^{0}, z^{0}\right)=\inf _{s \in \mathbb{R}^{n}}\left\{\varphi\left(x, y^{0}, s\right)+\frac{\left\|z^{0}-s\right\|^{2}}{2 \delta}\right\}=\varphi\left(x, y^{0}, s^{0}\right)+\frac{\left\|z^{0}-s^{0}\right\|^{2}}{2 \delta} .
\end{gathered}
$$

We then have

$$
\varphi\left(x, y_{k}, s_{k}\right)+\frac{\left\|z_{k}-s_{k}\right\|^{2}}{2 \delta} \leq \varphi\left(x, y_{k}, s^{0}\right)+\frac{\left\|z_{k}-s^{0}\right\|^{2}}{2 \delta} .
$$

Using (H5) we deduce

$$
-C_{1}\left\|s_{k}\right\|^{2}-C_{2}(x)+\frac{\left\|s_{k}\right\|^{2}}{2 \delta}-\frac{\left\|z_{k}\right\|^{2}}{2 \delta} \leq C_{1}\left\|s^{0}\right\|^{2}+C_{2}(x)+\frac{\left\|s^{0}\right\|^{2}}{2 \delta}+\frac{\left\|z_{k}\right\|^{2}}{2 \delta} .
$$

Taking $\delta<\frac{1}{4 C_{1}}$ we deduce that $s_{k}$ is bounded since $z_{k}$ is bounded. We can extract a subsequence denoted also $s_{k}$ such that $s_{k} \rightarrow \xi$ with $\xi \in \mathbb{R}^{n}$.

Passing to the limit in (8.20) we deduce

$$
\varphi\left(x, y^{0}, \xi\right)+\frac{\left\|z^{0}-\xi\right\|^{2}}{2 \delta} \leq \varphi\left(x, y^{0}, s^{0}\right)+\frac{\left\|z^{0}-s^{0}\right\|^{2}}{2 \delta},
$$

so $\xi=s^{0}$ since $s^{0}$ is the minimum of

$$
s \mapsto \varphi\left(x, y^{0}, s\right)+\frac{\left\|z^{0}-s\right\|^{2}}{2 \delta} .
$$

From the uniqueness of the minimum $s^{0}$, we deduce that all the subsequences of $s_{k}$ converge to $s^{0}$. We then have

$$
J_{x, y_{k}, \delta}\left(z_{k}\right) \rightarrow J_{x, y^{0}, \delta}\left(z^{0}\right)
$$


which gives from (8.18) the desired continuity for $\nabla_{z} \varphi_{\delta}$. Passing to the limit in (8.19) we deduce the continuity for $\varphi_{\delta}$.

The measurability of the function $x \mapsto \varphi_{\delta}(x, y, z)$ is immediate from the measurability of the function $x \mapsto \varphi(x, y, z)$ and the definition of $\varphi_{\delta}$. We now have for any fixed $y \in \mathbb{R}^{m}$, $z \in \mathbb{R}^{n}$

$$
\frac{\partial \varphi_{\delta}}{\partial z_{j}}(x, y, z)=\lim _{k \rightarrow \infty} k\left[\varphi_{\delta}\left(x, y, z+\frac{1}{k} e_{k}\right)-\varphi_{\delta}(x, y, z)\right] .
$$

Since the function $x \in \Omega \rightarrow k\left[\varphi_{\delta}\left(x, y, z+\frac{1}{k} e_{k}\right)-\varphi_{\delta}(x, y, z)\right]$ is measurable, then the function $x \rightarrow \frac{\partial \varphi_{\delta}}{\partial z_{j}}(x, y, z)$ is measurable. The last result (iv) is an immediate consequence of Proposition 8.2

We now give the main result of this section:

THEOREM 8.5. Let $u^{\varepsilon}$ in $\left(L^{2}(\Omega)\right)^{n}$, which two-scale converges to some $u$ in $\left(L^{2}(\Omega \times Y)\right)^{n}$, and $\varphi$ be such that $(H 1)-(H 5)$ hold. Then we have

$$
\liminf _{\varepsilon \rightarrow 0} \int_{\Omega} \varphi\left(x, \frac{x}{\varepsilon}, u^{\varepsilon}(x)\right) d x \geq \int_{\Omega} \int_{Y} \varphi((x, y, u(x, y)) d y d x .
$$

Proof. Let

$$
b=\liminf _{\varepsilon \rightarrow 0} \int_{\Omega} \varphi\left(x, \frac{x}{\varepsilon}, u^{\varepsilon}(x)\right) d x,
$$

so there exists a subsequence of $\varepsilon$, still denoted by $\varepsilon$, such that

$$
\int_{\Omega} \varphi\left(x, \frac{x}{\varepsilon}, u^{\varepsilon}(x)\right) d x \rightarrow b \quad \text { when } \varepsilon \rightarrow 0 .
$$

We also have

$$
\begin{aligned}
& \int_{\Omega} \varphi\left(x, \frac{x}{\varepsilon}, u^{\varepsilon}(x)\right) d x \geq \int_{\Omega} \varphi_{\delta}\left(x, \frac{x}{\varepsilon}, u^{\varepsilon}(x)\right) d x \\
& \geq \int_{\Omega} \varphi_{\delta}\left(x, \frac{x}{\varepsilon}, v_{0}\left(x, \frac{x}{\varepsilon}\right)\right) d x \\
&+\int_{\Omega} \nabla_{z} \varphi_{\delta}\left(x, \frac{x}{\varepsilon}, v_{0}\left(x, \frac{x}{\varepsilon}\right)\right) \cdot\left[u^{\varepsilon}(x)-v_{0}\left(x, \frac{x}{\varepsilon}\right)\right] d x \\
& \text { for any } v_{0} \in(\mathcal{D}(\Omega \times Y))^{n} .
\end{aligned}
$$

From Lemma 8.4 and using also Lemma 5.3 in [15], we deduce that

(1) $(x, y) \mapsto \varphi_{\delta}\left(x, y, v_{0}(x, y)\right)$ is in $L^{1}\left(\Omega, \mathcal{C}_{\#}(Y)\right)$,

(2) $(x, y) \mapsto \nabla_{z} \varphi_{\delta}\left(x, y, v_{0}(x, y)\right)$ is in $\left(L^{2}\left(\Omega, \mathcal{C}_{\#}(Y)\right)\right)^{n}$.

We can now pass to the limit, $\varepsilon \rightarrow 0$, in (8.21), using Theorem 2, p.40 of [16] and also Theorem 7, p.48 of [16] and that $u^{\varepsilon}$ two-scale converges to $u$, to get

$$
\begin{aligned}
b \geq & \int_{\Omega} \int_{Y} \varphi_{\delta}\left(x, y, v_{0}(x, y)\right) d y d x \\
& +\int_{\Omega} \int_{Y} \nabla_{z} \varphi_{\delta}\left(x, y, v_{0}(x, y)\right) \cdot\left[u(x, y)-v_{0}(x, y)\right] d y d x .
\end{aligned}
$$

Thus

$$
(u, b) \in \mathcal{D}_{v_{0}, \delta} \quad \forall \delta>0 \quad \text { and } \quad \forall v_{0} \in(\mathcal{D}(\Omega \times Y))^{n} .
$$


From Lemma 8.3 we deduce that

$$
(u, b) \in \operatorname{epi}\left(S_{0}^{\delta}\right) \quad \forall \delta>0,
$$

and from Proposition 8.3 we deduce that $(u, b) \in \operatorname{epi}\left(S_{0}\right)$. Thus

$$
b=\liminf _{\varepsilon \rightarrow 0} \int_{\Omega} \varphi\left(x, \frac{x}{\varepsilon} u^{\varepsilon}(x)\right) d x \geq S_{0}(u)=\int_{\Omega} \int_{Y} \varphi(x, y, u(x, y)) d y d x .
$$

\section{REFERENCES}

[1] R. Pit Mesure locale de la vitesse à l'interface solide-liquide simple: Glissement et rôle des interactions. Physics thesis, Univ. Paris XI, (1999).

[2] R. Pit, H. Hervet, L. Léger Direct experimental evidences for flow with slip at hexadecane solid interfaces. La revue de Métallurgie-CIT/Science, February (2001).

[3] G. Bayada, M. Boukrouche On a free boundary problem for the Reynolds equation derived from the Stokes system with Tresca boundary conditions. J. Math. Anal. Appl. (2003); 282, 212-231. MR2000339 (2004g:35237)

[4] M. Boukrouche, G. Łukaszewicz Asymptotic analysis of solutions of a thin film lubrication problem with Coulomb fluid-solid interface law. Internat. J. Engrg. Sci. (2003); 41, 521-537. MR 1954918 (2003j:76032)

[5] M. Boukrouche and G. Lukaszewicz On a lubrication problem with Fourier and Tresca boundary conditions. Math. Models Methods Appl. Sci. (2004); 14, (6), 913-941. MR2069499 (2005d:35277)

[6] M. Boukrouche, R. El Mir Asymptotic analysis of a non-Newtonian fluid in a thin domain with Tresca law. Nonlinear Anal. (2004), 59, no.1-2, 85-105. MR2092079 (2005f:35331)

[7] M. Boukrouche, F. Saidi Non-isothermal lubrication problem with Tresca fluid-solid interface law. Part I. To appear in Nonlinear Anal. Real World Applications.

[8] G. Duvaut, J.L. Lions Les inéquations en mécanique et en physique. Dunod, Paris, (1972). MR0464857 (57:4778)

[9] N. Benhaboucha, M. Chambat, I. Ciuperca Asymptotic behaviour of pressure and stresses in a thin film flow with a rough boundary. Quart. Appl. Math. (2005), 63, 369-400. MR2150781 (2006h:35207)

[10] G. Bayada, I. Ciuperca, M. Jai Homogenized elliptic equations and variational inequalities with oscillating parameters. Application to the study of thin flow behavior with rough surfaces. To appear in Nonlinear Anal.: Real World Applications.

[11] G. Buscaglia, I. Ciuperca, M. Jai Existence and uniqueness for several non-linear elliptic problems arising in lubrication theory. J. Differential Equations, 218, Issue 1, 1 November 2005, 187-215. MR2174972

[12] G. Buscaglia, I. Ciuperca and M. Jai On nano-scale hydrodynamic lubrication models. CRAS Mécanique, Vol. 333, Issue 6, June 2005, Pages 453-458.

[13] V. Girault, P.A. Raviart Finite Element Approximation of the Navier-Stokes Equations. Springer-Verlag, (1979). MR.0548867 (83b:65122)

[14] R. Temam Navier-Stokes Equations. Theory and Numerical Analysis. North-Holland, Amsterdam, (1979). MR0603444 (82b:35133)

[15] G. Allaire Homogenization and two-scale convergence. SIAM J. Math. Anal. (1992), 23, no.6, 1482-1518. MR.1185639 (93k:35022)

[16] D. Lukkassen, G. Nguetseng, P. Wall Two-scale convergence. Int. J. Pure Appl. Math. (2002), 2, no. 1, 35-86. MR1912819 (2003f:35019)

[17] I. Ekeland, R. Temam Analyse convexe et problèmes variationnels. Dunod 1974. MR0463993 $(57: 3931 \mathrm{a})$

[18] U. Hornung, W. Jäger, A. Mikelić Reactive transport through an array of cells with semipermeable membranes. RAIRO Modl. Math. Anal. Numér. (1994), 28, no.1, 59-94. MR1259268 (94m:76118)

[19] A. Bourgeat, A. Mikelic A Note on the Homogenization of Bingham Flow through a Porous Medium. J. Math. Pures et Appl., 72 (1993), 405-414. MR1228999 (94d:76085) 
[20] A. Bourgeat, A. Mikelic, S. Wright On the Stochastic Two-Scale Convergence in the Mean and Applications. J. Reine Angew. Math. 456 (1994), 19-51. MR1301450 (99b:35008)

[21] V. Barbu, T. Precupanu Convexity and Optimization in Banach Spaces. Mathematics and its applications. Ets. Acdemiei Bucuresti, Roumania (1986).

[22] H. Brézis Opérateurs Maximaux Monotones et semi-groupes de contractions dans les espaces de Hilbert. North-Holand, Mathematics Studies (1973). MR0348562 (50:1060) 